\title{
Phase-delay Induced Variation of Synchronization Bandwidth and Frequency Stability in a Micromechanical Oscillator
}

\section{Zhan Shi}

Zhejiang University

\section{Dong Pu}

Zhejiang University

\section{Xuefeng Wang}

Zhejiang University

Ronghua Huan ( $\square$ rhhuan@zju.edu.cn )

Zhejiang University https://orcid.org/0000-0003-4648-3805

\section{Zhuangde Jiang}

Xian Jlaotong University

\section{Xueyong Wei}

Xian Jiaotong University: Xi'an Jiaotong University

\section{Research Article}

Keywords: micromechanical oscillator, nonlinear dynamics, synchronization , feedback tuning , frequency stability

Posted Date: April 8th, 2021

DOl: https://doi.org/10.21203/rs.3.rs-394057/v1

License: (c) (i) This work is licensed under a Creative Commons Attribution 4.0 International License. Read Full License

Version of Record: A version of this preprint was published at Nonlinear Dynamics on August 7th, 2021. See the published version at https://doi.org/10.1007/s11071-021-06783-8. 


\title{
Phase-delay induced variation of synchronization bandwidth and frequency stability in a micromechanical oscillator
}

\author{
Zhan Shi · Dong Pu · Xuefeng Wang • Ronghua Huan* · Zhuangde \\ Jiang · Xueyong Wei*
}

Received: date / Accepted: date

\begin{abstract}
Phase feedback is commonly utilized to set up a synchronized MEMS oscillator for high performance sensor applications. It's a consensus that the synchronization region varies with phase delay with a 'Anti-U' mode within 0 to $\pi$ and phase delay is typically fixed on $\pi / 2$ to achieve maximum synchronization range and best frequency stability. In this paper, phase-delay induced variation of synchronization bandwidth and frequency stability in a micromechanical os-
\end{abstract}

*Corresponding author

Zhan Shi

Department of Mechanics, Key Laboratory of Soft Machines and Smart Devices of Zhejiang Province, Zhejiang University, Hangzhou, 310027, China

E-mail: zhanshi@zju.edu.cn

\section{Dong $\mathrm{Pu}$}

Department of Mechanics, Key Laboratory of Soft Machines and Smart Devices of Zhejiang Province, Zhejiang University, Hangzhou, 310027, China

E-mail: pd@zju.edu.cn

Xuefeng Wang

Department of Mechanics, Key Laboratory of Soft Machines and Smart Devices of Zhejiang Province, Zhejiang University, Hangzhou, 310027, China

Ronghua Huan

Department of Mechanics, Key Laboratory of Soft Machines and Smart Devices of Zhejiang Province, Zhejiang University, Hangzhou, 310027, China

E-mail: rhhuan@zju.edu.cn

Zhuangde Jiang

State Key Laboratory for Manufacturing Systems Engineering, Xi'An Jiaotong University, Xi'An, 710049, China

Xueyong Wei

State Key Laboratory for Manufacturing Systems Engineering, Xi'An Jiaotong University, Xi'An, 710049, China

E-mail: seanwei@mail.xjtu.edu.cn cillator is investigated analytically and experimentally. A self-sustained oscillator is built by applying phase feedback to an electrostatically actuated micro-beam resonator and synchronization phenomenon is observed after coupling it to a weak external periodic excitation. The analytical expression for predicting the synchronization bandwidth with phase delay is derived based on the dynamic model, from which three different types ('U', 'Anti-U' and 'M') of variation pattern of synchronization bandwidth are observed as feedback tuning. The variation of frequency stability along phase delay is also studied. The synchronization bandwidth and the frequency stability have exactly opposite variation pattern with phase delay in linear oscillators while they are totally consistent in nonlinear oscillators. Experimental tests in vacuum environment are carried out to validate the analytical observations. Our work presented here provides a precise way for achieving best performance of a synchronized MEMS oscillator in the sensor application.

Keywords micromechanical oscillator · nonlinear dynamics · synchronization · feedback tuning · frequency stability

\section{Introduction}

Microelectromechanical systems (MEMS) have increasingly attracted considerable interest due to their small size and low power consumption since the integrated circuit (IC) technology rise in 1990. Related investigations not only focus on fundamental questions, such as frequency locking[28,26,27], internal resonance[7,20, 41], mode coupling[4,29,9], pull-in[14,43], exit time[42], bilayer effect[17], etc., but also in tremendous potential applications including gas/mass sensing[12,34,33], 
filters[1], memory devices[39] and time keeping[3]. Due to the size effect, MEMS system is easily driven into nonlinear regime. In MEMS, nonlinearity can typically arise from external potentials[8], or from geometric deformati A typical phenomenon is that nonlinearity can enhance the synchronization behavior. Synchronization usually refers to the harmonization between the phases of at least two vibration systems. The earliest research on synchronization phenomenon can be traced back to the observation of the synchronization phenomenon of coupled pendulum by C. Huygens in 1673. When one of the vibration systems is a perturbation source which is generated by external signal generator, it shows a behavior that the oscillator's frequency will synchronously changing with the external perturbation frequency, which makes it a great choice to utilize this property as frequency sensing. Great quantity of works have been done in synchronization, such as synchronization in chaotic systems[18,19], superharmonic synchronization [6, 24], subharmonic synchronization[40,25,38], etc. Lots of works have shown the advantages of synchronization as sensing such as reduce the phase noise[36,15], enhance the frequency stability[10,30], etc. Meanwhile, the time delay in system are also considered in energy harvesting $[5$, 32], traffic flow[13] and as well, synchronization of Micromechanical systems[10]. Most importantly, enhancing the synchronization bandwidth has become a hot topic. Some researchers find that nonlinearity in the oscillator can be exploited to enhance the synchronization bandwidth[2]. Their experimental and analytical results show that nonlinearities are the key determinants in the nonlinear regime in a self-sustained micromechanical oscillator. Phase feedback is commonly utilized to keep a self-sustained micro-oscillator. Previous studies show that the synchronization bandwidth varies with the phase delay in a 'Anti-U' pattern. In theroretical and experimental studies the phase delay is always fixed at $\pi / 2$ to obtain a maximum amplitude $[37,22,35,21]$, or to get a maximum synchronization bandwidth $[2,10]$. Nevertheless, after in-depth analysis and experimental verification, we get some new findings.

In this paper, the variation of synchronization region and frequency stability with phase delay in a micromechanical oscillator is investigated, and three different variation patterns('U', 'Anti-U' and 'M') of synchronization bandwidth are observed analytically and experimentally. The novelty and contributions of our work lie in the following: 1) the analytical expression for predicting the synchronization bandwidth with phase delay in micromechanical oscillators is derived, from which three variation patterns ('U', 'Anti-U' and 'M') of synchronization bandwidth with phase delay are observed as feedback tuning; 2) the synchronization band- (a)

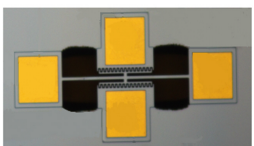

(b)

(c)
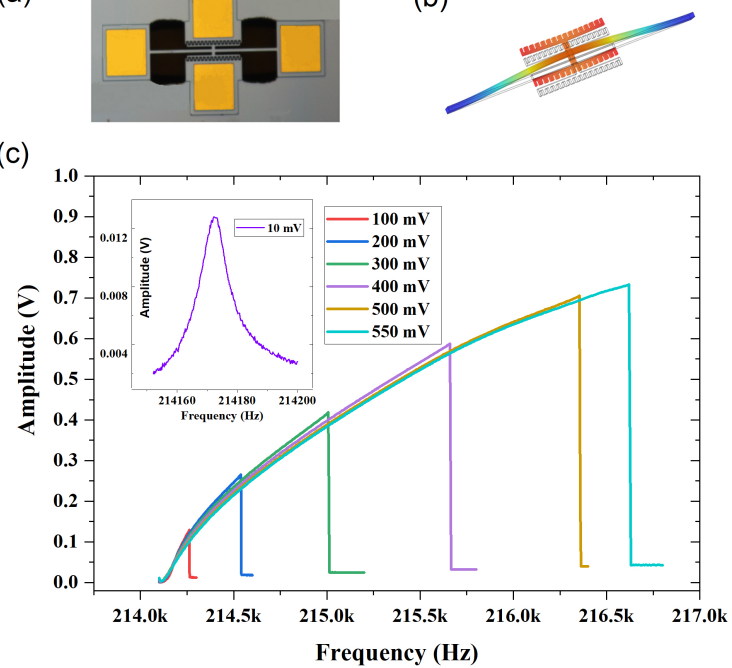

Fig. 1 Characteristics of the micro-beam resonator. a SEM image of the micro-resonator. The gray part is monocrystalline silicon, the golden part is gold electrode, and the black part is hollow; b Primary modal shape simulation of the oscillator in COMSOL; c Open-loop amplitude-frequency responses of the micro-resoantor under $V_{D C}=20 \mathrm{~V}$ and $V_{f}$ varying from $100 \mathrm{mV}$ to $550 \mathrm{mV}$. Inset shows the open-loop response for $V_{f}$ equals $10 \mathrm{mV}$, in which the micro-resonator works in linear regime and corresponding measured quality factor $Q \sim 20000$.

width and the frequency stability have exactly opposite variation pattern along phase delay in linear oscillators while they are consistent in nonlinear oscillators; 3 ) our work presented enriches the synchronization phenomenon and provides a precise way for achieving best performance of a synchronized MEMS oscillator in the sensor application.

\section{Theoretical analysis}

\subsection{Basic features of the micro-resonator}

A clamped-clamped(C-C) micromechanical beam resonator with dimensions $482 \mu \mathrm{m}$ long, $12 \mu \mathrm{m}$ wide, and $25 \mu \mathrm{m}$ thick, respectively, as shown in Fig. 1(a), is studied in this paper. This micromechanical resonator is electrostatically actuated to vibrate at its primary modal by applying a combination of constant voltage $V_{D C}$ and dynamic voltage $V_{f}$ on the mid electrostatic comb. The primary modal shape simulation worked by Comsol of the micro-resonator is shown in Fig.1(b). Figure 1(c) shows the open-loop amplitude-frequency responses of the resonator for a fixed $V_{D C}\left(V_{D C}=20 \mathrm{~V}\right)$ and various $V_{f}$ from $10 \mathrm{mV}$ to $550 \mathrm{mV}$. Inset displays the resonator vibrates under small driving strength $V_{f}$ $=10 \mathrm{mV}$. It is seen that for small driving strength, the 
resonator vibrates with small amplitude in its linear regime, and the resonator exhibits strong nonlinearity when driving strength increasing.

Based on the Euler-Bernoulli beam carrying a concentrated mass in the mid-span, the governing equation of the transverse deflection $\hat{w}(u, t)$ for the microbeam, is written as[11],

$$
\begin{aligned}
& E I \frac{\partial^{4} \hat{w}}{\partial \hat{u}^{4}}+\left(\rho S+m_{c} \delta\left(\hat{u}-\frac{L}{2}\right)\right) \frac{\partial^{2} \hat{w}}{\partial \hat{t}^{2}}+\hat{c} \frac{\partial \hat{w}}{\partial \hat{t}} \\
& =\frac{E S}{2 L} \int_{0}^{L}\left(\frac{\partial \hat{w}}{\partial \hat{u}}\right)^{2} \mathrm{~d} \hat{u} \frac{\partial^{2} \hat{w}}{\partial \hat{u}^{2}}+\hat{F}\left(\hat{w}, \hat{\omega} \hat{t}, \hat{\omega}_{s} \hat{t}\right) \delta\left(\hat{u}-\frac{L}{2}\right)
\end{aligned}
$$

where $E$ is the Young's modulus, $I$ and $S$ are the moment of inertia and area of the cross section. $\hat{u}$ is the location along the beam, $\hat{t}$ is time, $L$ and $\rho$ are the total length and material density, respectively. $\hat{c}$ is the viscous damping per unit length and $m_{c}$ is the mass of the middle comb structure. $\hat{F}$ is the combination of the applied electrostatic feedback sustaining force $\hat{F}_{0}$ and the synchronizing perturbation force $\hat{F}_{s}$. Introducing the following nondimensional variables $w=\frac{\hat{w}}{b}, x=$ $\frac{\hat{u}}{L}, t=\hat{t} \sqrt{\frac{E I}{\rho S L^{4}}}$, Eq. (1) can be simplified as,

$$
\begin{aligned}
& \frac{\partial^{4} w}{\partial x^{4}}+\left(1+\bar{m} \delta\left(x-\frac{1}{2}\right)\right) \frac{\partial^{2} w}{\partial t^{2}}+c \frac{\partial w}{\partial t} \\
& \quad=\alpha \int_{0}^{1}\left(\frac{\partial w}{\partial x}\right)^{2} \mathrm{~d} x \frac{\partial^{2} w}{\partial x^{2}}+F\left(w, \omega t, \omega_{s} t\right) \delta\left(x-\frac{1}{2}\right)
\end{aligned}
$$

The C-C beam subjected to the following boundary conditions,

$$
\begin{array}{cl}
w(0, t) & =0, \quad w(L, t)=0, \\
\frac{\partial w(0, t)}{\partial x} & =0, \quad \frac{\partial w(L, t)}{\partial x}=0 .
\end{array}
$$

Considering that in our experiments the $\mathrm{AC}$ driving voltage is set much lower than the DC bias voltage $\left(V_{a c} \ll V_{D C}\right)$ to minimize the effect from harmonic excitations (such as subharmonic synchronization), and the perturbaiton strength $V_{f}$ is much lower than the driving voltage $\left(V_{s} \ll V_{a c}\right)$. When synchronization arises, retaining the first three orders after Taylor expansion, $F$ is simplified as $[31,16]$,

$$
\begin{aligned}
& F\left(w, \omega t, \omega_{s} t\right)=\frac{L^{3} \gamma}{E I d}\left(4 w+8 w^{3}\right) \\
& +\frac{2 L^{3} \gamma V_{a c}}{E I d V_{D C}}\left(\frac{d^{2}}{g a}+1+2 w+3 w^{2}+4 w^{3}\right) \sin (\omega t) \\
& +\frac{2 L^{3} \gamma V_{s}}{E I d V_{D C}}\left(\frac{d^{2}}{g a}+1\right) \sin \left(\omega_{s} t\right)
\end{aligned}
$$

where $w_{s}$ is the synchronization perturbation frequency. The first mode is dominant in our experiments when the resonator works near its natural frequency. By using the Galerkin decomposition method, the solution of Eq. (2) is approximated as,

$w(x, t)=x(t) n(t)$,

where $x(t)$ is the time varying first mode coordinate, $n(t)$ is the first modal shape function, for a $C-C$ beam, $n(t)$ usually has the form,

$$
\begin{aligned}
n(t)= & -\frac{\bar{m}_{w_{n}}}{4}\left[\left(\frac{\sinh \sqrt{w}_{n} x}{\cosh \sqrt{w}_{n} L}-\frac{\sin \sqrt{w}_{n} x}{\cos \sqrt{w}_{n} L}\right)\right. \\
& \left.-2 U\left(x-\frac{1}{2}\right)\left[\sinh \sqrt{w}_{n}\left(x-\frac{1}{2}\right)-\sin \sqrt{w}_{n}\left(x-\frac{1}{2}\right)\right]\right]
\end{aligned}
$$

where $w_{n}$ is the natural frequency. The dominant first mode dynamic model is obtained,

$$
\begin{aligned}
M \frac{\mathrm{d}^{2} x}{\mathrm{~d} t^{2}}+C \frac{\mathrm{d} x}{\mathrm{~d} t}+ & K_{1} x+K_{3} x^{3} \\
& =F_{f b} \sin (\omega t)+F_{\text {syn }} \sin \left(\omega_{s} t\right)
\end{aligned}
$$

where,

$$
\begin{aligned}
& M=\int_{0}^{1} n(t)^{2} \varepsilon(x) \mathrm{d} x, K_{1}=\int_{0}^{1} n(t)^{\prime \prime 2} \mathrm{~d} x-\frac{4 \gamma L^{3}}{E I d} n^{2}\left(\frac{1}{2}\right), \\
& C=c \int_{0}^{1} n(t)^{2} \mathrm{~d} x, K_{3}=\alpha\left(\int_{0}^{1} n(t)^{\prime 2} \mathrm{~d} x\right)^{2}-\frac{8 \gamma L^{3}}{E I d} n(t)^{2}\left(\frac{1}{2}\right), \\
& F_{f b}=\frac{2 L^{3} \gamma V_{a c}}{E I d V_{D C}} n(t)\left(\frac{1}{2}\right)\left(\frac{d^{2}}{g a}+1\right), \\
& F_{\text {syn }}=\frac{2 L^{3} \gamma V_{s}}{E I d V_{D C}} n(t)\left(\frac{1}{2}\right)\left(\frac{d^{2}}{g a}+1\right) .
\end{aligned}
$$

where $M, C, K_{1}, K_{3}, F_{f b}$ and $F_{\text {syn }}$ are the effective mass, damping coefficient, linear mechanical stiffness, cubic mechanical stiffness, feedback force and external synchronization force, respectively. The feedback control force $F_{f b}=F_{0} \cos \left(\phi_{\text {ins }}+\phi_{d e}\right)$ is generated by a close-loop feedback circuit to compensate the energy dissipation and make the resonator a self-sustained oscillator. $F_{0}$ is the magnitude of the feedback force, $\phi_{\text {ins }}$ is the instantaneous phase of the oscillator, $\phi_{d e}$ denotes the phase delay between the oscillator and feedback force. $F_{\text {syn }}=F_{\text {syn }} \cos \left(\Omega_{\text {syn }} t\right)$ is the perturbation force injected into the self-oscillation oscillator. It is known that if the injected signal frequency $\Omega_{\text {syn }}$ is approaching the resonator's oscillation frequency $\Omega$ in a small range, synchronization occurs. After redefining time units $(t \sqrt{K / M} \rightarrow t)$, the normalized motion equation becomes

$$
\ddot{x}+Q^{-1} x+x+\beta x^{3}=f_{0} \cos \left(\phi+\phi_{0}\right)+f_{s} \cos \left(\Omega_{s} t\right)
$$


where $Q=\frac{\sqrt{K M}}{C}$ denotes the quality factor, which represents the rate of energy loss, $\beta=\frac{K_{3}}{K_{1}}, f_{0}=\frac{F_{f b}}{K_{1}}$, $f_{s}=\frac{F_{s y n}}{K_{1}}$ and $\Omega_{s}=\frac{\omega_{s}}{\sqrt{K_{1} / M}}$ are the normalized Duffing nonlinearity, the amplitude of feedback force, weak periodic perturbation force, normalized perturbation frequency, respectively.

\subsection{Synchronization bandwidth with phase delay}

The analytical solution of Eq. (9) can be solved using the harmonic solution,

$$
\begin{gathered}
x=A \cos (\phi) \\
\dot{x}=-A \Omega \sin (\phi)
\end{gathered}
$$

Substituting Eq. (10) into Eq. (9), we have

$$
\begin{array}{r}
-\mathrm{A} \Omega^{2} \cos (\phi)-\mathrm{Q}^{-1} \mathrm{~A} \Omega \\
\sin (\phi)+\mathrm{A} \cos (\phi)+\beta \mathrm{A}^{3} \cos ^{3}(\phi) \\
=f_{0} \cos \left(\phi+\phi_{0}\right)+f_{s} \cos \left(\Omega_{s} t\right)
\end{array}
$$

In the synchronization case, due to the frequency locking effect, the oscillation frequency $\Omega$ of the resonator is exactly equal to the perturbation frequency $\Omega_{s}$, i.e., $\Omega=\Omega_{s}$. Then, we have $\Omega_{s} t=\phi+\phi_{s}$. Equation (11) becomes

$$
\begin{array}{r}
-A \Omega^{2} \cos (\phi)-Q^{-1} A \Omega \sin (\phi)+A \cos (\phi)+\beta A^{3} \cos ^{3}(\phi) \\
=f_{0} \cos (\phi) \sin \left(\phi_{0}\right)-f_{0} \sin (\phi) \cos \left(\phi_{0}\right) \\
+f_{s} \cos (\phi) \sin \left(\phi_{s}\right)-f_{s} \sin (\phi) \cos \left(\phi_{s}\right)
\end{array}
$$

Neglecting higher order harmonic contributions, we have the approximation expression[23]

$\cos ^{3}(\phi) \approx \frac{3}{4} \cos (\phi)$

Substituting Eq. (13) into Eq. (12) and separating terms in Eq. (12) proportional to $\cos (\phi)$ and $\sin (\phi)$, respectively, we get two equations which can be merged into a single equation in the complex domain

$$
\begin{aligned}
& \left\{\left(1-\Omega^{2}\right) \mathrm{A}+\frac{3}{4} \beta A^{3}-f_{0} \cos \left(\phi_{0}\right)\right\} \\
& +\left\{\mathrm{A} \Omega \mathrm{Q}^{-1}+f_{0} \sin \left(\phi_{0}\right)\right\} i=f_{s} e^{-i \phi_{s}}
\end{aligned}
$$

Firstly, we consider the dynamic characteristics of the self-sustained resonator without the external perturbation, i.e., $f_{0} \neq 0, f_{s}=0$. In this case, two separate equations can be obtained from Eq. (14) as

$$
\begin{aligned}
\left(1-\Omega^{2}\right) A+\frac{3}{4} \beta A^{3}-f_{0} \cos \left(\phi_{0}\right) & =0 \\
A \Omega Q^{-1}+f_{0} \sin \left(\phi_{0}\right) & =0
\end{aligned}
$$

$\Omega_{0}$ and $A_{0}$ are obtained by solving Eq. (15) and (16)

$$
\begin{gathered}
A_{0}=\frac{Q f_{0} \sin \left(\phi_{0}\right)}{\Omega_{0}} \\
\Omega_{0}=\frac{1}{\sqrt{2}}\left(1+\left(1+3 \beta Q^{2} f_{0}^{2} \sin ^{2}\left(\phi_{0}\right)\right)^{\frac{1}{2}}\right)^{\frac{1}{2}}
\end{gathered}
$$

Here, $\Omega_{0}$ and $A_{0}$ are vibration frequency and amplitude of the self-sustained oscillator. When the external perturbation is applied, i.e. $f_{s} \neq 0, f \neq 0$, the frequency and amplitude of the oscillator will deviation from self-oscillation frequency. For weak perturbation, (i.e. $f_{s} \ll f_{0}$ ), the variation of frequency $\delta \Omega$ and amplitude $\delta A$ of the oscillator are expected to be small compared to $\Omega_{0}$ and $A_{0}$. Introducing the perturbative parameter $\epsilon=f_{s} / f_{0} \ll 1$, and rewriting $\delta \Omega$ and $\delta A$ as $\epsilon \delta \Omega$ and $\epsilon \delta A$, respectively. The instantaneous frequency and amplitude are

$\Omega=\Omega_{0}+\epsilon \delta \Omega, A=A_{0}+\epsilon \delta A$

Substituting Eq. (19) into Eq. (14) ,by neglecting higher order terms, we get

$$
\begin{aligned}
\left\{\left(1-\Omega_{0}\right)^{2}\right. & \left.+\frac{9}{4} \beta A_{0}^{2}-i \Omega_{0} Q^{-1}\right\} \delta A \\
& -\left\{2 A_{0} \Omega_{0}+i A_{0} Q^{-1}\right\} \delta \Omega=f_{0} e^{-\left(i \phi_{s}\right)}
\end{aligned}
$$

The modulus of the complex equation Eq. (20) satisfies

$N_{r}^{2}+N_{i}^{2}=f_{0}^{2}$

where $N_{r}=\left\{\left(1-\Omega_{0}^{2}\right)+\frac{9}{4} \beta A_{0}^{2}\right\} \delta A-2 A_{0} \Omega_{0} \delta \Omega$ and $N_{i}=Q^{-1}\left(A_{0} \delta \Omega+\Omega_{0} \delta A\right)$ are real and imaginary terms of Eq. (20). Treat Eq. (21) as a quadratic equation of $\delta A$

$$
\begin{array}{r}
\left\{\left(\left(1-\Omega_{0}\right)^{2}+\frac{9}{4} \beta A_{0}^{2}\right)^{2}+\frac{\Omega_{0}^{2}}{Q^{2}}\right\} \delta A^{2} \\
+\left\{\frac{2 A_{0} \Omega_{0} \delta \Omega}{Q^{2}}-4 A_{0} \Omega_{0}\left(\left(1-\Omega_{0}\right)^{2}+\frac{9}{4} \beta A_{0}^{2}\right)\right\} \delta A \\
+\left\{\left(4 \Omega_{0}^{2}+\frac{1}{Q^{2}}\right) A_{0}^{2} \delta \Omega^{2}-f_{0}^{2}\right\}=0
\end{array}
$$

To ensure the real solution of Eq. (22), the following expression satisfied

$$
\begin{array}{r}
\left\{\frac{2 A_{0} \Omega_{0} \delta \Omega}{Q^{2}}-4 A_{0} \Omega_{0}\left(\left(1-\Omega_{0}\right)^{2}+\frac{9}{4} \beta A_{0}^{2}\right)\right\}^{2}- \\
4\left\{\left(\left(1-\Omega_{0}\right)^{2}+\frac{9}{4} \beta A_{0}^{2}\right)^{2}+\frac{\Omega_{0}^{2}}{Q^{2}}\right\} \\
\left\{\left(4 \Omega_{0}^{2}+\frac{1}{Q^{2}}\right) A_{0}^{2} \delta \Omega^{2}-f_{0}^{2}\right\} \geq 0
\end{array}
$$



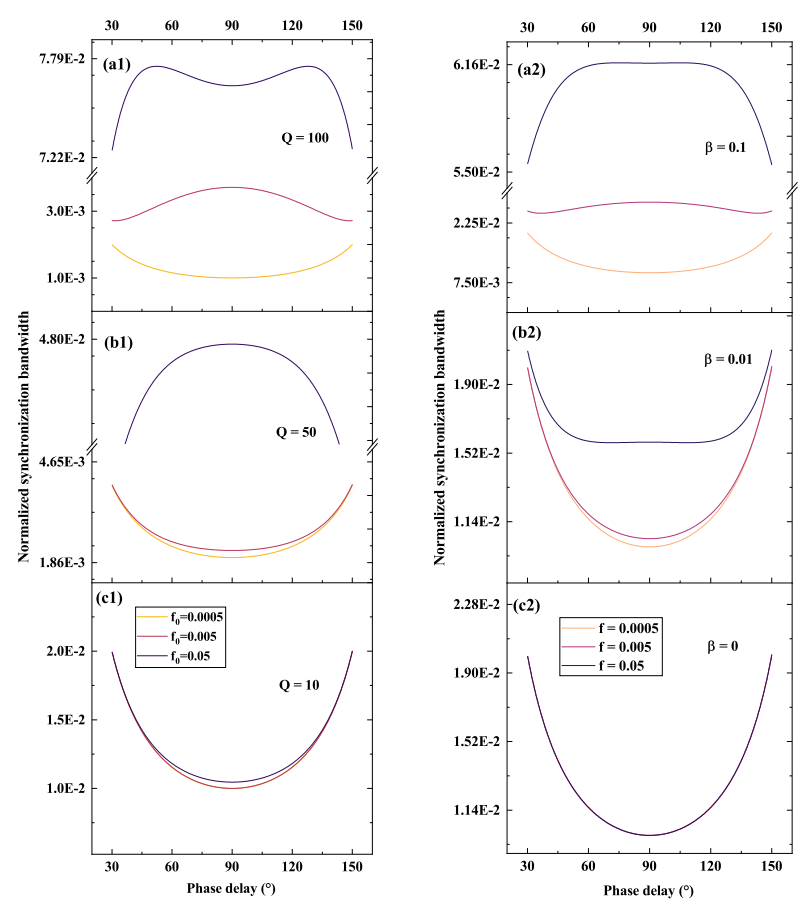

Fig. 2 Predicted synchronization bandwidth behavior obtained from Eq.(25) under feedback force equals 0.0005(yellow line), 0.005(purple line) and 0.05(black line). a1 $Q=100$; b1 $Q=50$; c1 $Q=10$ are under fixed $\beta=0.1$, a2 $\beta=0.1$; b2 $\beta=0.01$; c2 $\beta=0$ are under fixed $Q=10$.

Equation.(23) holds under the synchronization condition. Solving Eq.(23) the limit frequency deviation $\delta \Omega_{c}$ is determined, which leads to a synchronization range $\left(-\delta \Omega_{c}, \delta \Omega_{c}\right)$

$\left|\delta \Omega_{c}\right| \leq \epsilon \frac{Q}{A_{0}}\left(1-\frac{\left(144 \beta A_{0}^{2}+64-\frac{16}{Q^{2}}\right) \Omega_{0}^{2}}{\left(4+4 \Omega_{0}^{2}+9 \beta A_{0}^{2}\right)^{2}}\right)^{\frac{1}{2}}$

Thus, the analytical expression of full synchronization bandwidth is obtained as $\mathcal{B}=2 \delta \Omega_{c}$, i.e.,

$\mathcal{B}=2 \frac{Q f_{s}}{f_{0} A_{0}}\left(1-\frac{\left(144 \beta A_{0}^{2}+64-\frac{16}{Q^{2}}\right) \Omega_{0}^{2}}{\left(4+4 \Omega_{0}^{2}+9 \beta A_{0}^{2}\right)^{2}}\right)^{\frac{1}{2}}$

where $\Omega_{0}$ and $A_{0}$ are determined by Eq. (17) and (18). From Eq. (25) we can see that the synchronization bandwidth is nonlinear function of nonlinearity $\beta$ and quality factor $Q$, which would be distinct in different oscillators. We plot the variation form of synchronization bandwidth along with phase delay under different feedback forces in Fig. 2, it can seen that the quality factor $Q$ and nonlinearity $\beta$ has similar effects on the variation forms of synchronization bandwidth along with phase delay under different strength of feedback force $f_{0}$. For an oscillator with lower quality factor $Q$, its synchronization bandwidth along with phase delay exhibits a
'U' form, the minimum synchronization bandwidth appears at phase delay equals $\pi / 2$, and different feedback force $f_{0}$ almost has no effect on the size of synchronization bandwidth, which is similar with a linear oscillator shown in Fig. 2(c2) $(\beta=0)$. While for larger quality factor $Q=50$ (Fig. 2(b1)) and relatively larger nonlinearity $\beta=0.01$ (Fig. 2(b2)), the synchronization bandwidth also presents a ' $U$ ' form under small strength of feedback force. As $f_{0}$ increases to 0.05 (black line), the synchronizaiton form begins to bulge from the bottom and evolves toward a 'Anti-U' form. Most intrestingly, for a large quality factor or a strong nonlinearity under strong feedback force(Black lines in Fig. 2(a1) and Fig. $2(\mathrm{a} 2))$, the form of synchronizaiton bandwidth exhibits a 'M' form, the synchronization bandwidth emerges two maximum values and a minimum values. From Eqs. (17) and (18) we can see that the oscillator's self-oscillation amplitude is govered by the quality factor $Q$, nonlinearity $\beta$, feedback force $f_{0}$ and phase delay $\phi_{0}$ (the perturbation strength $f_{s}$ is small enough, i.e. $\frac{\left|A-A_{0}\right|}{A_{0}} \approx 0$ ). For a given nonlinearity $\beta$, the smaller quality $Q$ and smaller feedback force $f_{0}$ will lead to a smaller amplitude $A_{L}$, thus the nonlinearity term $\beta A_{L}^{3}$ can be neglected compared to linearity term $A_{L}$, the oscillator will exhibits as linear. As for a larger quality factor $Q_{H}$ and nonlinearity $\beta_{H}$, the self oscillation amplitude $A_{H}$ will become considerable, so the nonlinearity term $\beta A_{H}^{3}$ can be comparable with linear term $A_{H}$, the nonlinearity start to work, even play a leading role. Above results remind us that for different oscillators, we can obtain the maximum synchronization bandwidth by adjusting the phase delay and the feedback force simultaneously, which can be achieved in our experimental tests.

\subsection{Variation forms of synchronization region}

For a given oscillator driven in a stable test environment, we just consider the effect of the feedback force and phase delay. The synchronization bandwidth $\mathcal{B}$ is a nonlinear function of phase delay $\phi_{0}$ as shown in Eq. (25). After ignoring small amounts, $\frac{d \mathcal{B}}{d \phi_{0}}=0$ leads to the following two solutions

$\phi_{0,1}=\frac{\pi}{2}, \quad \phi_{0,2}=\arcsin \left(\frac{1}{Q \sqrt{\beta} f_{0}}\right)$

where $\phi_{0,1}$ is a fixed extrenal point at $\pi / 2$, which is a traditional result in previous studies[10]. $\phi_{0,2}$ is an additional dynamic one, which is motivated by the nonlinearity $\beta$ of the oscillator. Figure 3 shows the $\phi_{0,1}$ and $\phi_{0,2}$ vary with feedback force $f_{0}$ in different oscillators. 


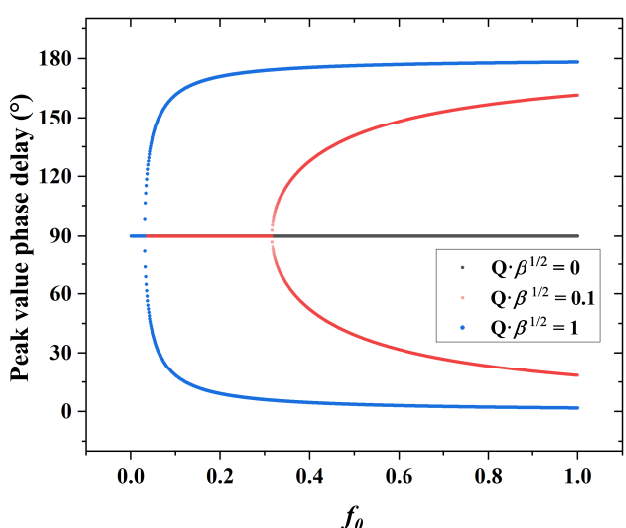

Fig. 3 Phase delay of synchronization bandwidth's peak value varies with feedback force $f_{0}$ in oscillator with different quality factor $Q$ and nonlinearity $\beta$. Where black dots: $Q \cdot \beta^{1 / 2}=0 ;$ red dots: $Q \cdot \beta^{1 / 2}=0.1 ;$ blue dots: $Q \cdot \beta^{1 / 2}=1$

The black $\operatorname{dots}(Q \cdot \sqrt{\beta}=0)$ represent a linear oscillator, where the peak value of synchronization bandwidth always exists at stable phase delay $\pi / 2$, which is not affected by the strength of feedback force $f_{0}$. The red $\operatorname{dots}(Q \cdot \sqrt{\beta}=0.1)$ represent a oscillator with weakly nonlinearity, as the strength of feedback force is weak, the phase delay of peak value fixed at $\pi / 2$. However, when the strength of feedback force getting larger, the single phase delay split into two symmetrical points about $90^{\circ}$. And when the nonlinearity becomes strong(blue dots, where $Q \cdot \sqrt{\beta}=1$ ), the bifurcation point emerges at smaller feedback force $f_{0}$. For convenience of experimental verification in a specific oscillator with approximately changeless nonlinearity and stable atmospheric pressure (a stable quality factor $Q$ ), we fixed $Q \cdot \sqrt{\beta}=1$ and plot the synchronization bandwidth vary with phase delay under different feedback forces.

Figure 4 displays the synchronization region $\mathcal{B}$ varying with phase delay $\phi_{0}$ for different feedback strength $f_{0}$, calculated from Eq. (25) with parameters $Q=10$, $\beta=0.1, \epsilon=f_{s} / f_{0}=0.1$. Interestingly, three different types of variation pattern of the synchronization bandwidth with the phase delay is observed.

\subsection{1 'U' form}

For small feedback strength, the synchronization bandwidth varies with the phase delay with a ' $U$ ' form as shown in Fig. 4b(I). The minimum synchronization bandwidth is achieved when $\phi_{0}=\pi / 2$. This is because, for small feedback strength, the oscillator vibrates in the linear regime with a very small amplitude (i.e., $\beta=0$ ), which leads to the reduced expression of synchroniza-

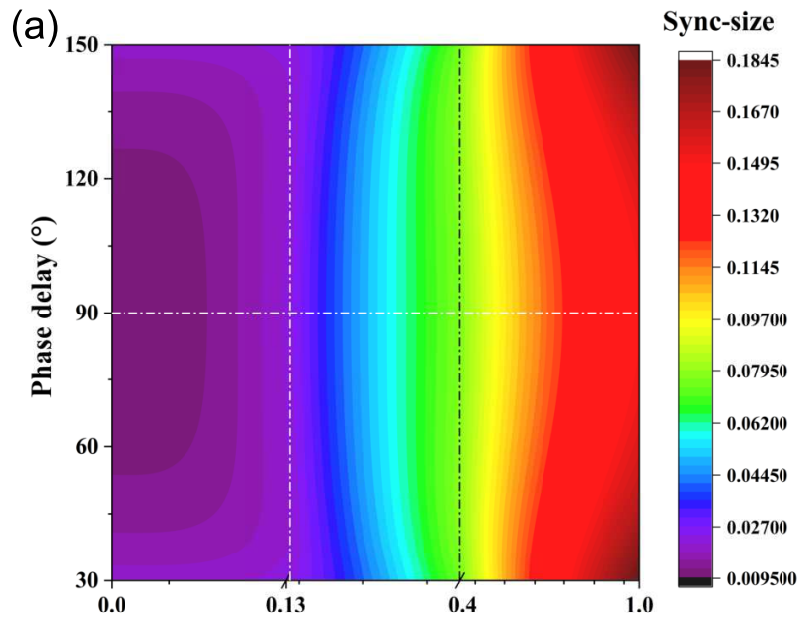

(b)

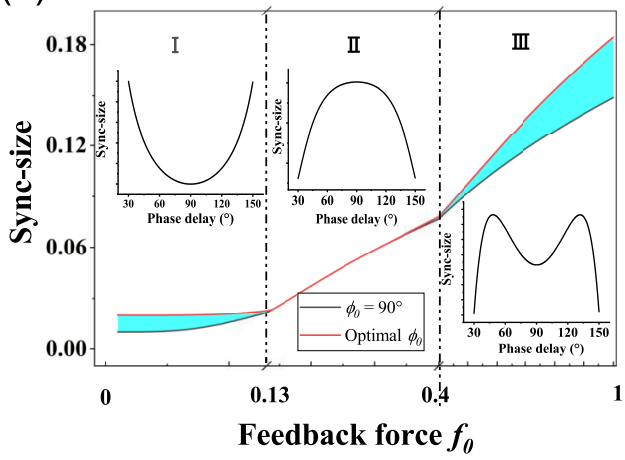

Fig. 4 Predicted synchronization bandwidth behavior obtained from Eq.(25). a The synchronization bandwidth varies with phase delay for different feedback forces $f_{0}$ obtained from Eq.(25), where $\mathrm{Q}=10, \beta=0.1, \epsilon=0.1, f_{0}$ varies from 0.01 to 1 with steps 0.02 ; $\mathbf{b}$ Red line: maximum synchronization bandwidth at optimal $\phi_{0}$ proposed by Eq. (25) at phase delay varies from $30^{\circ}$ to $90^{\circ}$. Black line: synchronization bandwidth from previous[10] by fixing $\phi_{0}=\pi / 2$. Insets show the evolution of variation pattern of synchronization bandwidth with phase delay as the feedback force $f_{0}$ increasing, where regime $I$ : 'U' pattern; regime $I I$ : 'Anti-U' pattern; regime $I I I$ : 'M' pattern.

tion bandwidth from Eq. (25)

$\mathcal{B}=\frac{f_{s}}{Q f_{0} \sin \left(\phi_{0}\right)}=\epsilon \cdot\left(\frac{1}{Q \sin \left(\phi_{0}\right)}\right)$

Only fixed extremal point $\phi_{0,1}$ is found in Eq. (27) and dynamic one $\phi_{0,2}$ disappears. Equation (27) gives a ' $U$ ' mode curve of synchronization bandwidth $\mathcal{B}$ with phase delay $\phi_{0}$ within $(0, \pi)$. Synchronization bandwidth $\mathcal{B}$ decreases first and increases again as $\phi_{0}$ increasing from 0 to $\pi$. There exists a minimum value at $\phi_{0}=\pi / 2$.

\subsection{2 'Anti-U' form}

As feedback strength increasing, a 'Anti-U' pattern variation of the synchronization bandwidth of the resonator 
is observed as shown in Fig. 4b(II). In this case, the synchronization bandwidth has a maximum value as phase delay $\phi_{0}=\pi / 2$, which is consistent with the previous studies refer to [10]. Since for a relatively large feedback strength, the nonlinear term is not negligible and there exists two extreme points $\phi_{0,1}$ and $\phi_{0,2}$ as seen in Eq. (26). However, for small feedback strength, the second extreme point $\phi_{0,2}$ is very close to the first one $\phi_{0,1}$, which leads to the 'Anti-U' form curve in Fig. 4b(II).

\subsection{3 ' $M$ ' form}

As the feedback strength increased to a much larger value, $\phi_{0,2}$ will separate from $\phi_{0,1}$ due to the decrease of $\frac{1}{Q \sqrt{\beta} f_{0}}$ in Eq. (26). Two peaks will exist in the synchronization bandwidth curves, which lead to a ' $\mathrm{M}$ ' shape curve as shown in Fig. $4 \mathrm{~b}(I I I)$. As the feedback strength further increased, the values of these two peaks of the synchronization bandwidth curves get larger and two peaks grow apart from each other. The colored region in Fig.4(b) displays the difference between the maximum synchronization bandwidth obtained from expression (25) (black line) and the synchronization bandwidth at a fixed phase delay $\phi_{0}=\pi / 2$ (red line) for varying feedback strength. It can be seen that only when the strength of feedback $f_{0}$ is in a certain region (regime $I I$ ), the maximum synchronization bandwidth achieved at $\phi_{0}=\pi / 2$. This means that the previous method of synchronization bandwidth calculation is only suitable for a small range of $f_{0}$.

\section{Experimental results}

In this section, experimental tests are carried out to validate the analytical results above. The experimental setup is shown in Fig. 5. The micro single beam resonator is clamped to clamped(C-C) and works near its natural frequency actuated by a combination of dynamic voltage $V_{f}$ and bias voltage $V_{D C}$. The motional signal is converted to electrical signal and detected by the HF2LI lock-in amplifier after pre-amplified by the differential circuit. The vacuum chamber can create a vacuum environment to reduce damping. The lock-in amplifier HF2LI with a built-in Phase Locked Loop(PLL) can output and detect signals simultaneously. Meanwhile, it can accurately tune the phase of the feedback signal. The signal generator can output synchronous disturbance signal to generate synchronization. All the measurements are tested in the vacuum chamber at a pressure below $0.1 \mathrm{~Pa}$ at room temperature. The measured oscillation frequency of the free running self-sustaine oscillator is $214500 \mathrm{~Hz}$ and the measured quality factor $\mathrm{Q} \sim 20000$ under the condition of $V_{f}$ equals to $10 \mathrm{mV}$.

\subsection{Synchronization region}

The synchronization regions under different excitation voltages are measured in this section. We set $V_{D C}=$ $20 \mathrm{~V}$ and $V_{f}$ fixed at a specific voltage when tuning the phase delay $\phi_{0}$ from $30^{\circ}$ to $90^{\circ}$ with a step $5^{\circ}$. A periodic perturbation signal is injected into the oscillator by a signal generator. Set $V_{D C}=20 \mathrm{~V}$ and feedback voltage strength $V_{f}=400 \mathrm{mV}$. The oscillation frequencies of the resonator varying with the perturbation frequency when sweeps up (red solid line) and down (black solid line) are shown in Fig. 6(a). It is obvious that there is a region (orange region) where the oscillation frequency is captured by the perturbation frequency. We extract the values of upper limit, central frequency and lower limit, then calculated the synchronization bandwidth at each test. Figure. 6 (b) Figure. 6 (e) show the synchronization region varying with phase delay for different feedback strength $V_{f}$. For small feedback strength $(10 \mathrm{mV}$ in Fig. 6(b)), the bandwidth of synchronization region decreases monotonously with phase delay. It is expected that the bandwidth has a minimum value when $\phi_{0}=\pi / 2$, which is consistent with the analytically predicted ' $U$ ' case. As the feedback strength increased to $200 \mathrm{mV}$ (Fig. 6(c)), the bandwidth is observed to be a monotonous increasing function of phase delay, the calcuated synchronization bandwidth agrees with the analytically predicted 'Anti-U' case. Further increasing feedback strength to $500 \mathrm{mV}$ and $550 \mathrm{mV}$, the second extreme point except $\phi_{0}=\pi / 2$ appears as shown in Figs. 6(d) and (e), which validates the analytical 'M' case. The peak value gets larger and corresponding phase delay reduced from $60^{\circ}$ to $55^{\circ}$ as feedback strength increased from $500 \mathrm{mV}$ to $550 \mathrm{mV}$ as shown in Fig. 6(f), which is consistent with the analytical results in Fig. $4 \mathrm{a}(I I I)$.

\subsection{Frequency stability}

Experiments are performed to investigate the effect of phase delay on the frequency stability of the oscillator. Allan deviation is an effective indicator for frequency stability evaluation, which defined by the frequency fluctuations averaged over an integration time $\tau$. Figure 7 plots the Allan deviation at integration time $\tau=1 \mathrm{~s}$ as functions of phase delay for different feedback strength. The whole Allan deviation curves during integration time $\tau \in(0.2,100)$ can be found in Fig. 8. The frequency stability of the oscillator varies with the phase delay and variation pattern of the fredquency stability is observed evolutionary as feedback strength increasing. For small feedback force, the resonator vibrates in linear regime. The Allan deviation 


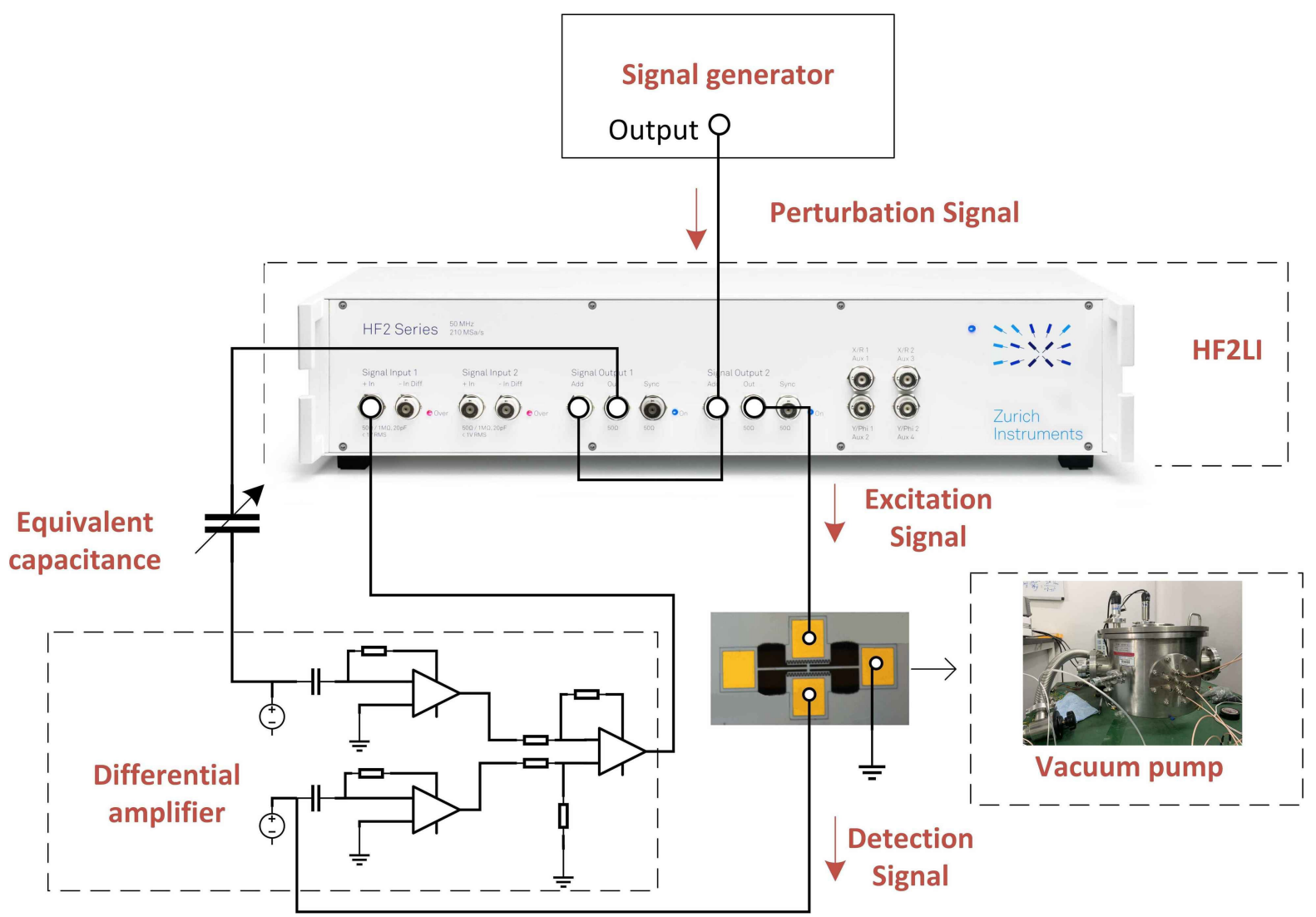

Fig. 5 The schematic of the closed-loop system. The micro-resonator is $482 \mu \mathrm{m}$ long, $12 \mu \mathrm{m}$ wide, $25 \mu \mathrm{m}$ thick respectively and located in a vacuum below $0.1 P a$ at room temperature. The drive signal is generated by HF2LI and added to the oscillator, while the oscillation signal is reinjected into the HF2LI to create a closed-loop system after amplified by differential circuit.

is a monotonically decreasing function of phase delay, and the frequency stability (red line in Fig. 7) and synchronization region (green line in Fig. 6(e)) varies with the phase delay with same pattern. The best frequency stability corresponds to the minimum synchronization bandwidth. However, interestingly, for larger feedback strength (in nonlinear regime), the frequency stability and synchronization region varies with the phase delay with totally opposite pattern. The best frequency stability and maximum synchronization bandwidth can be achieved simultaneously. This means that for linear oscillator better frequency stability is achieved for the price of the bandwidth of synchronization region, while for nonlinear oscillator there exists a good balance between the synchronization bandwidth and frequency stability.

We can see that for longer integration time shown in Fig. 8, the Allan deviation gradually getting smaller, which means that the frequency stability of the oscillation frequency works in its synchronization regime gradually tends to the stability of the signal generator as the phase changes from $0^{\circ}$ to $90^{\circ}$. For a long time, the frequency stability of synchronization tends to approach the generator's stability, this reminds us that for appli- cation, a stable synchronization source will bring better detection results.

\section{Conclusion}

In this paper, phase delay induced variation of synchronization bandwidth and frequency stability in a micromechanical oscillator subject to feedback tuning was investigated. The expression for predicting the synchronization bandwidth with phase delay is derived, from which an additional dynamic extreme point $\phi_{0,2}$ is motivated by nonlinearity expect the traditional one $\phi_{0,1}=\pi / 2$, which leads to three different variation patterns ('U', 'Anti-U' and 'M') of synchronization bandwidth. The evolution of the synchronization bandwidth alters the distribution of frequency stability with phase delay. For linear oscillator, the synchronization bandwidth and the frequency stability have exactly opposite variation pattern along phase delay, which implies better frequency stability will be achieved for the price of the bandwidth of synchronization region. However, in nonlinear oscillators, the synchronization bandwidth and the frequency stability are totally consistent, and 
(a)

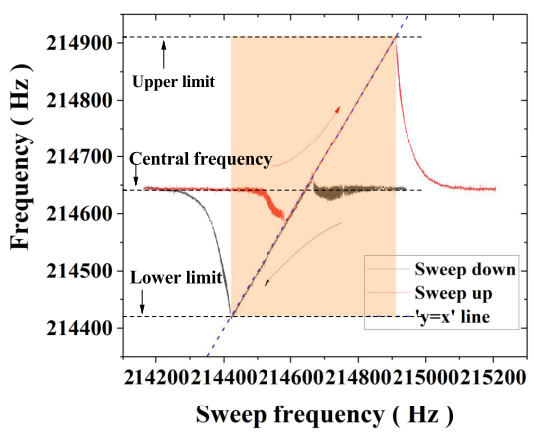

(d)

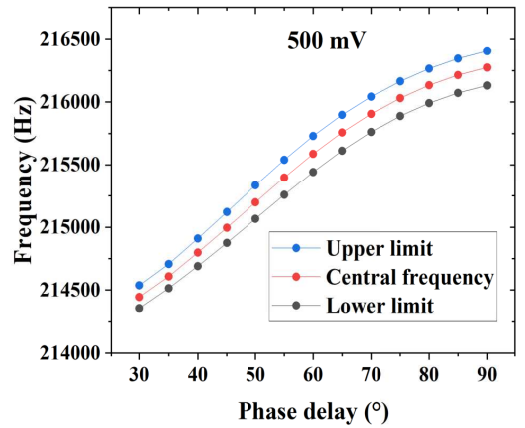

(b)

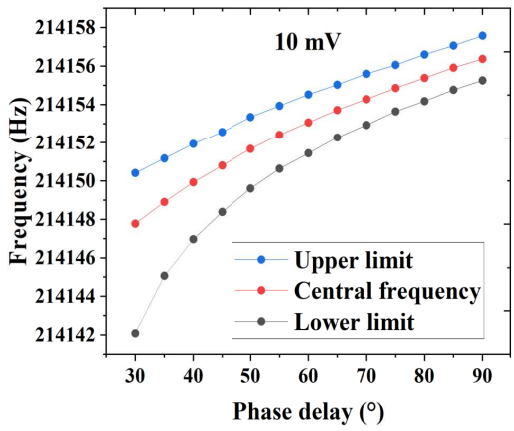

(e)

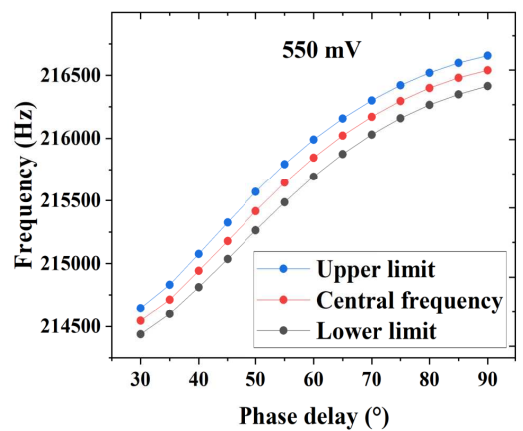

(c)

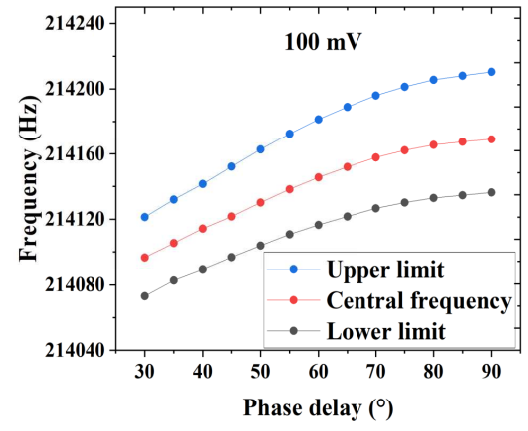

(f)

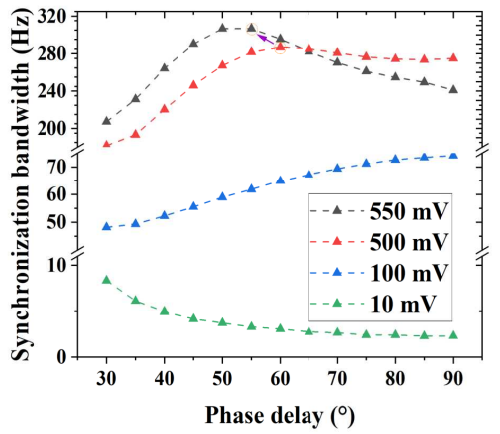

Fig. 6 Measured synchronization region. a Measured synchronization region under sweep up and sweep down perturbation at phase delay equals $60^{\circ}$ and feedback force exciting voltage $V_{f}=400 \mathrm{mV}$. The red line and the black line are the oscillation frequency varying as the perturbation frequency sweep and down, respectively, and the orange region is the synchronization region at this condition. $\mathbf{b}, \mathbf{c}, \mathbf{d}, \mathbf{e}$ are measured synchronization region under a fixed $V_{D C}=20 \mathrm{~V}$ and vary $V_{f}$, where $\mathbf{b}\left(V_{f}\right.$ $\left.=10 \mathrm{mV}, V_{s} / V_{f}=0.1\right), \mathbf{c}\left(V_{f}=100 \mathrm{mV}, V_{s} / V_{f}=0.1\right), \mathbf{d}\left(V_{f}=500 \mathrm{mV}, V_{s} / V_{f}=0.02\right), \mathbf{e}\left(V_{f}=550 \mathrm{mV}, V_{s} / V_{f}=0.02\right)\left(V_{s}\right.$ is the perturbation strength), where the blue line, red line and black line in $\mathbf{b}, \mathbf{c}, \mathbf{d}$, e are measured upper limit, central frequency and lower limit, respectively; $\mathbf{f}$ The measured synchronization bandwidth at $V_{f}$ equals $10 \mathrm{mV}, 100 \mathrm{mV}, 500 \mathrm{mV}$ and $550 \mathrm{mV}$, respectively. The purple arrow between two orange circles which are the peak values of synchronization bandwidth at $V_{D C}=500 \mathrm{mV}$ and $V_{D C}=550 \mathrm{mV}$ represents the movement of the peak value from $60^{\circ}$ to $55^{\circ}$ as $V_{f}$ varies from $500 \mathrm{mV}$ to $550 \mathrm{mV}$.

there exists a good balance between the synchronization bandwidth and frequency stability. The synchronization bandwidth evolution presented here provides a precise way for achieving best performance of a synchronized MEMS oscillator in the sensor application. We highlight that these results are applicable not only to MEMS resonator but also to any type of synchronized oscillator utilizing feedback.

\section{Acknowledgments}

This work was financially supported by the National Key Research and Development Program of China
(2018YFB2002303), National Natural Science Foundation of China (11772293, 52075432) and Key research and development program of Shaanxi Province (2018ZDCXL-GY-02-03).

\section{Conflict of interest}

The authors declare that they have no conflict of interest. 


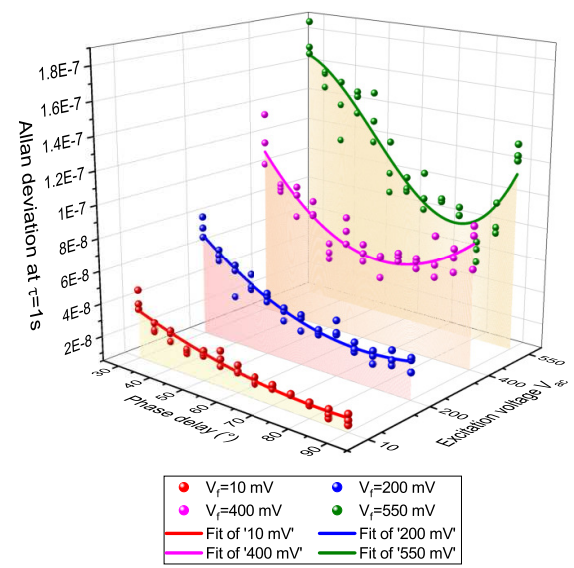

Fig. 7 Measured Allan deviation at integration time $\tau=1 \mathrm{~s}$ from experimental self-oscillation frequency at a fixed $V_{D C}$ $=20 \mathrm{~V}$ and $V_{f}$ equals $10 \mathrm{mV}, 200 \mathrm{mV}, 400 \mathrm{mV}$ and $550 \mathrm{mV}$ respectively, where scatters are measured results and real line are the fit line of scatters. Red scatter $\left(V_{f}=10 \mathrm{mV}\right)$ shows Allan deviation decrease as phase delay vary from $30^{\circ}$ to $90^{\circ}$ in linear regime, which has same variation pattern with synchronization region (green line in Fig. 3). better frequency stability is achieved for the price of the bandwidth of synchronization region. Blue scatter $\left(V_{f}=200 \mathrm{mV}\right)$, Purple $\operatorname{scatter}\left(V_{f}=400 \mathrm{mV}\right)$ and green $\operatorname{scatter}\left(V_{f}=550 \mathrm{mV}\right)$ show the Allan deviation varies with phase delay for nonlinear oscillator, which have totally opposite variation pattern with synchronization region. At the same time, as the feedback force strength increases, the most stable point of the selfoscillation frequency also decreases from $90^{\circ}$ to $0^{\circ}$. There exists a good balance between the synchronization bandwidth and frequency stability.

\section{References}

1. Al Hafiz, M.A., Kosuru, L., Hajjaj, A.Z., Younis, M.I.: Highly tunable narrow bandpass mems filter. IEEE Transactions on Electron Devices 64(8), 3392-3398 (2017)

2. Antonio, D., Czaplewski, D.A., Guest, J.R., López, D., Arroyo, S.I., Zanette, D.H.: Nonlinearity-induced synchronization enhancement in micromechanical oscillators. Physical review letters 114(3), 034103 (2015)

3. Antonio, D., Zanette, D.H., López, D.: Frequency stabilization in nonlinear micromechanical oscillators. Nature communications 3(1), 1-6 (2012)

4. Asadi, K., Yu, J., Cho, H.: Nonlinear couplings and energy transfers in micro-and nano-mechanical resonators: intermodal coupling, internal resonance and synchronization. Philosophical Transactions of the Royal Society A: Mathematical, Physical and Engineering Sciences 376(2127), 20170141 (2018)

5. Belhaq, M., Ghouli, Z., Hamdi, M.: Energy harvesting in a mathieu-van der pol-duffing mems device using time delay. Nonlinear Dynamics 94(4), 2537-2546 (2018)

6. Buonomo, A., Kennedy, M.P., Schiavo, A.L.: On the synchronization condition for superharmonic coupled qvcos. IEEE Transactions on Circuits and Systems I: Regular Papers 58(7), 1637-1646 (2011)

7. Chen, Y.s., Yang, C.x., Wu, Z.q., Chen, F.q.: 1: 2 internal resonance of coupled dynamic system with quadratic and cubic nonlinearities. Applied Mathematics and Mechanics 22(8), 917-924 (2001)

8. Cleland, A.N., Roukes, M.L.: A nanometre-scale mechanical electrometer. Nature 392(6672), 160-162 (1998)

9. Hajjaj, A.Z., Hafiz, M.A., Younis, M.I.: Mode coupling and nonlinear resonances of mems arch resonators for bandpass filters. Scientific reports 7, 41820 (2017)

10. Huan, R., Pu, D., Wang, X., Wei, X.: Effects of phase delay on synchronization in a nonlinear micromechanical oscillator. Applied Physics Letters 114(23), 233501 (2019)

11. Huan, R., Pu, D., Wei, X.: Phase switch in the stochastic response of a micromechanical beam resonator. Acta Mechanica 229(5), 2177-2187 (2018)

12. Jaber, N., Ilyas, S., Shekhah, O., Eddaoudi, M., Younis, M.I.: Multimode mems resonator for simultaneous sensing of vapor concentration and temperature. IEEE Sensors Journal 18(24), 10145-10153 (2018)

13. Jin, Y., Hu, H.: Stabilization of traffic flow in optimal velocity model via delayed-feedback control. Communications in Nonlinear Science and Numerical Simulation 18(4), 1027-1034 (2013)

14. Krylov, S., Seretensky, S.: Higher order correction of electrostatic pressure and its influence on the pull-in behavior of microstructures. Journal of Micromechanics and Microengineering 16(7), 1382 (2006)

15. Lebrun, R., Jenkins, A., Dussaux, A., Locatelli, N., Tsunegi, S., Grimaldi, E., Kubota, H., Bortolotti, P., Yakushiji, K., Grollier, J., et al.: Understanding of phase noise squeezing under fractional synchronization of a nonlinear spin transfer vortex oscillator. Physical review letters 115(1), 017201 (2015)

16. Li, L., Zhang, Q., Wang, W., Han, J.: Nonlinear coupled vibration of electrostatically actuated clamped-clamped microbeams under higher-order modes excitation. Nonlinear Dynamics 90(3), 1593-1606 (2017)

17. Liu, R., Wang, L.: Nonlinear forced vibration of bilayer van der waals materials drum resonator. Journal of Applied Physics 128(14), 145105 (2020)

18. Ma, J., Wu, F., Alsaedi, A., Tang, J.: Crack synchronization of chaotic circuits under field coupling. Nonlinear Dynamics 93(4), 2057-2069 (2018)

19. Mahmoud, G.M., Mahmoud, E.E.: Complete synchronization of chaotic complex nonlinear systems with uncertain parameters. Nonlinear Dynamics 62(4), 875-882 (2010)

20. Mangussi, F., Zanette, D.H.: Internal resonance in a vibrating beam: a zoo of nonlinear resonance peaks. PloS one 11(9), e0162365 (2016)

21. Matheny, M.H., Grau, M., Villanueva, L.G., Karabalin, R.B., Cross, M., Roukes, M.L.: Phase synchronization of two anharmonic nanomechanical oscillators. Physical review letters 112(1), 014101 (2014)

22. Mestrom, R., Fey, R., Nijmeijer, H.: On phase feedback for nonlinear mems resonators. In: 2007 IEEE International Frequency Control Symposium Joint with the 21st European Frequency and Time Forum, pp. 765-770. IEEE (2007)

23. Nayfeh, A.H., Mook, D.T.: Nonlinear oscillations. John Wiley \& Sons (2008)

24. Niu, J., Li, X., Xing, H.: Superharmonic resonance of fractional-order mathieu-duffing oscillator. Journal of Computational and Nonlinear Dynamics 14(7) (2019)

25. Oberreiter, L., Seifert, U., Barato, A.C.: Stochastic discrete time crystals: Entropy production and subharmonic synchronization. Physical Review Letters 126(2), 020603 (2021) 

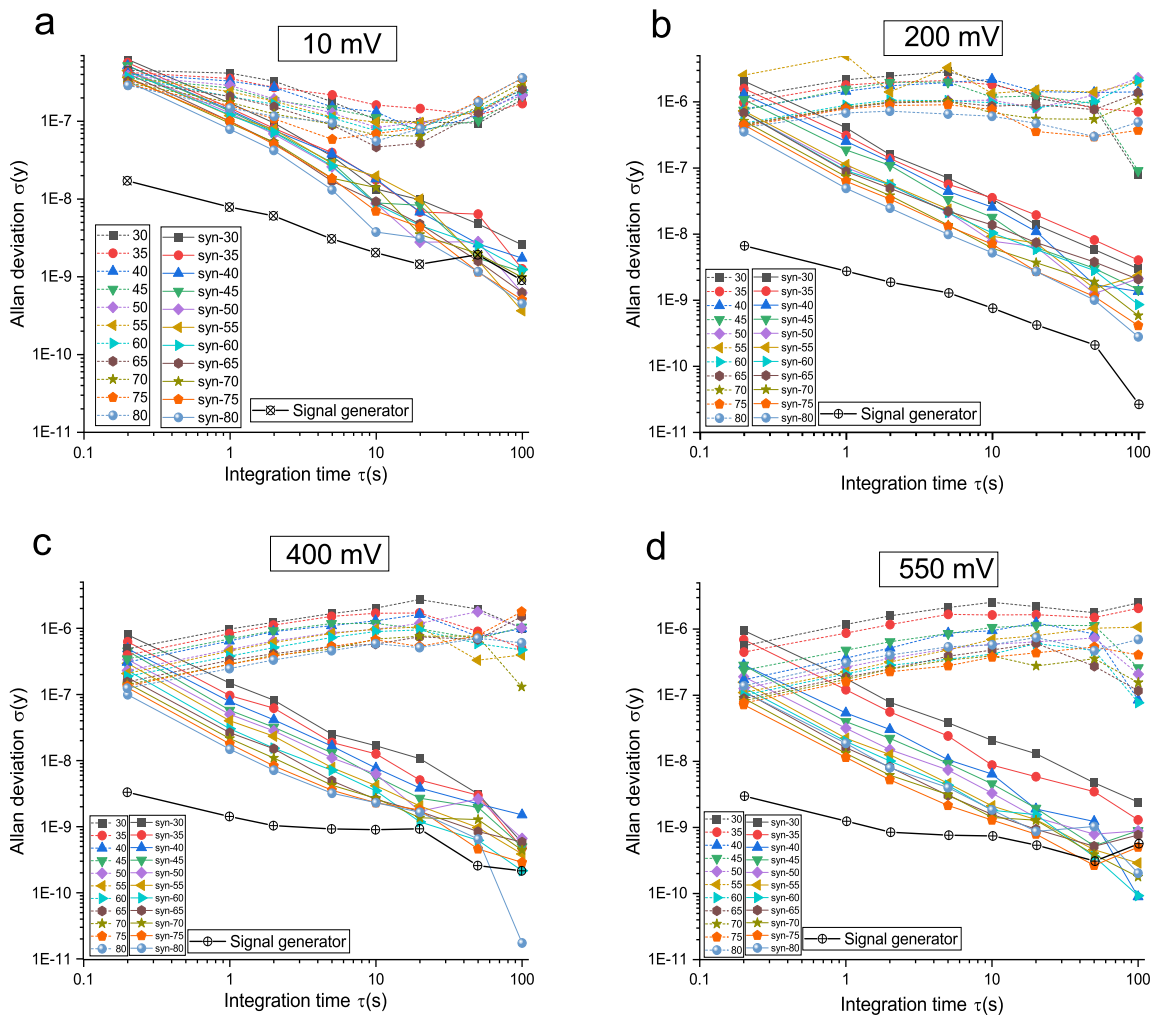

Fig. 8 Measured Allan deviation. Measured Allan deviation of oscillation frequency before and after synchronization at different phase delays and exciting voltages. The dashed and solid lines are measured Allan deviation before and after synchronization respectively. The thick black solid line is the Allan deviation of signal generator. Where $\mathbf{a} V_{f}=10 \mathrm{mV} ; \mathbf{b} V_{f}=200$ $m V ; \mathbf{c} V_{f}=400 m V ; \mathbf{d} V_{f}=550 m V$.

26. Pandey, M., Aubin, K., Zalalutdinov, M., Reichenbach, R.B., Zehnder, A.T., Rand, R.H., Craighead, H.G.: Analysis of frequency locking in optically driven mems resonators. Journal of Microelectromechanical systems 15(6), 1546-1554 (2006)

27. Pandey, M., Rand, R., Zehnder, A.: Perturbation analysis of entrainment in a micromechanical limit cycle oscillator. Communications in Nonlinear Science and Numerical Simulation 12(7), 1291-1301 (2007)

28. Pandey, M., Rand, R.H., Zehnder, A.T.: Frequency locking in a forced mathieu-van der pol-duffing system. In: International Design Engineering Technical Conferences and Computers and Information in Engineering Conference, vol. 48027, pp. 893-903 (2007)

29. Pandit, M., Zhao, C., Sobreviela, G., Mustafazade, A., Du, S., Zou, X., Seshia, A.A.: Closed-loop characterization of noise and stability in a mode-localized resonant mems sensor. IEEE transactions on ultrasonics, ferroelectrics, and frequency control 66(1), 170-180 (2018)

30. Pu, D., Huan, R., Wei, X.: Frequency stability improvement for piezoresistive micromechanical oscillators via synchronization. AIP Advances 7(3), 035204 (2017)

31. Pu, D., Yang, P., Wang, X., Huan, R., Jiang, Z., Wei, $\mathrm{X}$.: Anomalous amplitude-frequency dependence in a micromechanical resonator under synchronization. Nonlinear Dynamics 103(1), 467-479 (2021)

32. Richard, J.P.: Time-delay systems: an overview of some recent advances and open problems. automatica 39(10), 1667-1694 (2003)
33. Roukes, M.L., Hanay, M.S., Kelber, S., Naik, A.: Singleprotein nanomechanical mass spectrometry in real time (2016). US Patent 9,347,815

34. Sage, E., Brenac, A., Alava, T., Morel, R., Dupré, C., Hanay, M.S., Roukes, M.L., Duraffourg, L., Masselon, C., Hentz, S.: Neutral particle mass spectrometry with nanomechanical systems. Nature communications 6(1), $1-5(2015)$

35. Sato, M., Hubbard, B., Sievers, A., Ilic, B., Czaplewski, D., Craighead, H.: Observation of locked intrinsic localized vibrational modes in a micromechanical oscillator array. Physical review letters 90(4), 044102 (2003)

36. Shoshani, O., Heywood, D., Yang, Y., Kenny, T.W., Shaw, S.W.: Phase noise reduction in an mems oscillator using a nonlinearly enhanced synchronization domain. Journal of Microelectromechanical Systems 25(5), 870-876 (2016)

37. Sun, B., Zhao, C., Sobreviela-Falces, G., Du, S., Han, F., Zou, X., Seshia, A.: Enhanced frequency stability in a non-linear mems oscillator employing phase feedback. In: 2017 IEEE 30th International Conference on Micro Electro Mechanical Systems (MEMS), pp. 1115-1117. IEEE (2017)

38. Umemura, W., Ozeki, Y., Fujita, K., Fukui, K., Itoh, K.: Subharmonic synchronization of picosecond lasers for stimulated raman scattering microscopy. In: CLEO: Science and Innovations, p. CThF5. Optical Society of America (2011)

39. Uranga, A., Verd, J., Marigó, E., Giner, J., MuñózGamarra, J., Barniol, N.: Exploitation of non-linearities 
in cmos-nems electrostatic resonators for mechanical memories. Sensors and Actuators A: Physical 197, 88-95 (2013)

40. Velichko, A., Belyaev, M., Putrolaynen, V., Perminov, V., Pergament, A.: Thermal coupling and effect of subharmonic synchronization in a system of two vo2 based oscillators. Solid-State Electronics 141, 40-49 (2018)

41. Wang, X., Huan, R., Zhu, W., Pu, D., Wei, X.: Frequency locking in the internal resonance of two electrostatically coupled micro-resonators with frequency ratio 1 : 3 . Mechanical Systems and Signal Processing 146, 106981

42. Xu, Y., Li, H., Wang, H., Jia, W., Yue, X., Kurths, J.: The estimates of the mean first exit time of a bistable system excited by poisson white noise. Journal of Applied Mechanics 84(9) (2017)

43. Zhang, W.M., Yan, H., Peng, Z.K., Meng, G.: Electrostatic pull-in instability in mems/nems: A review. Sensors and Actuators A: Physical 214, 187-218 (2014) 
Figures

(a)

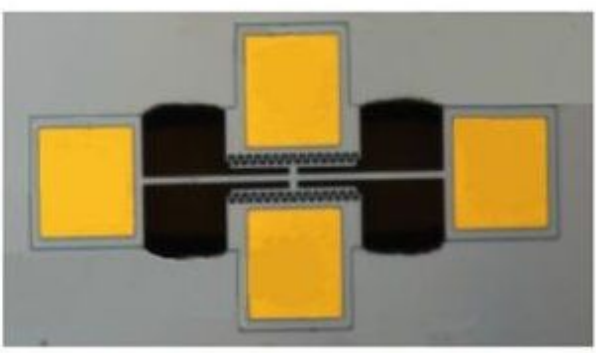

(b)

(c)

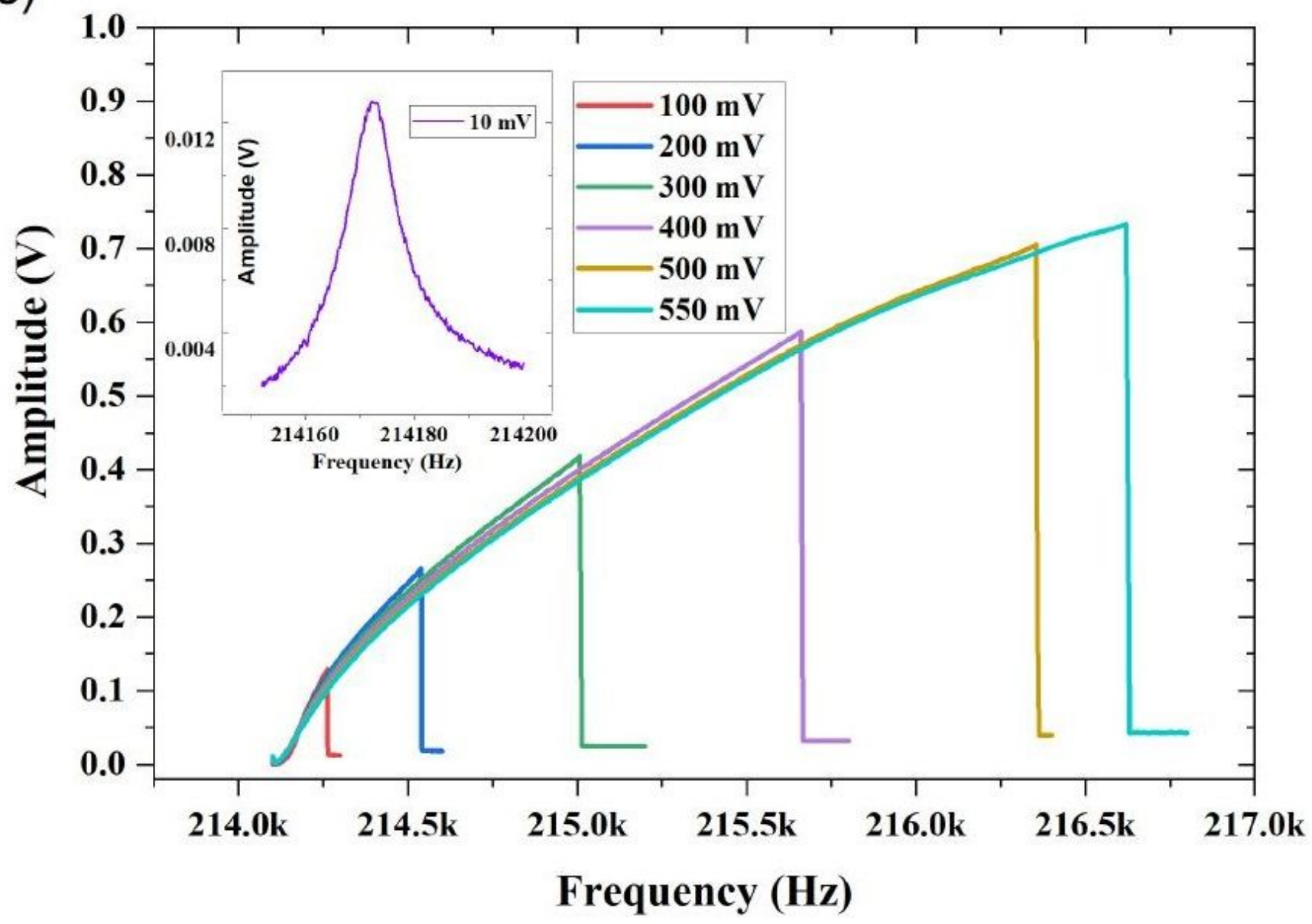

Figure 1

Please see the Manuscript PDF file for the complete figure caption 

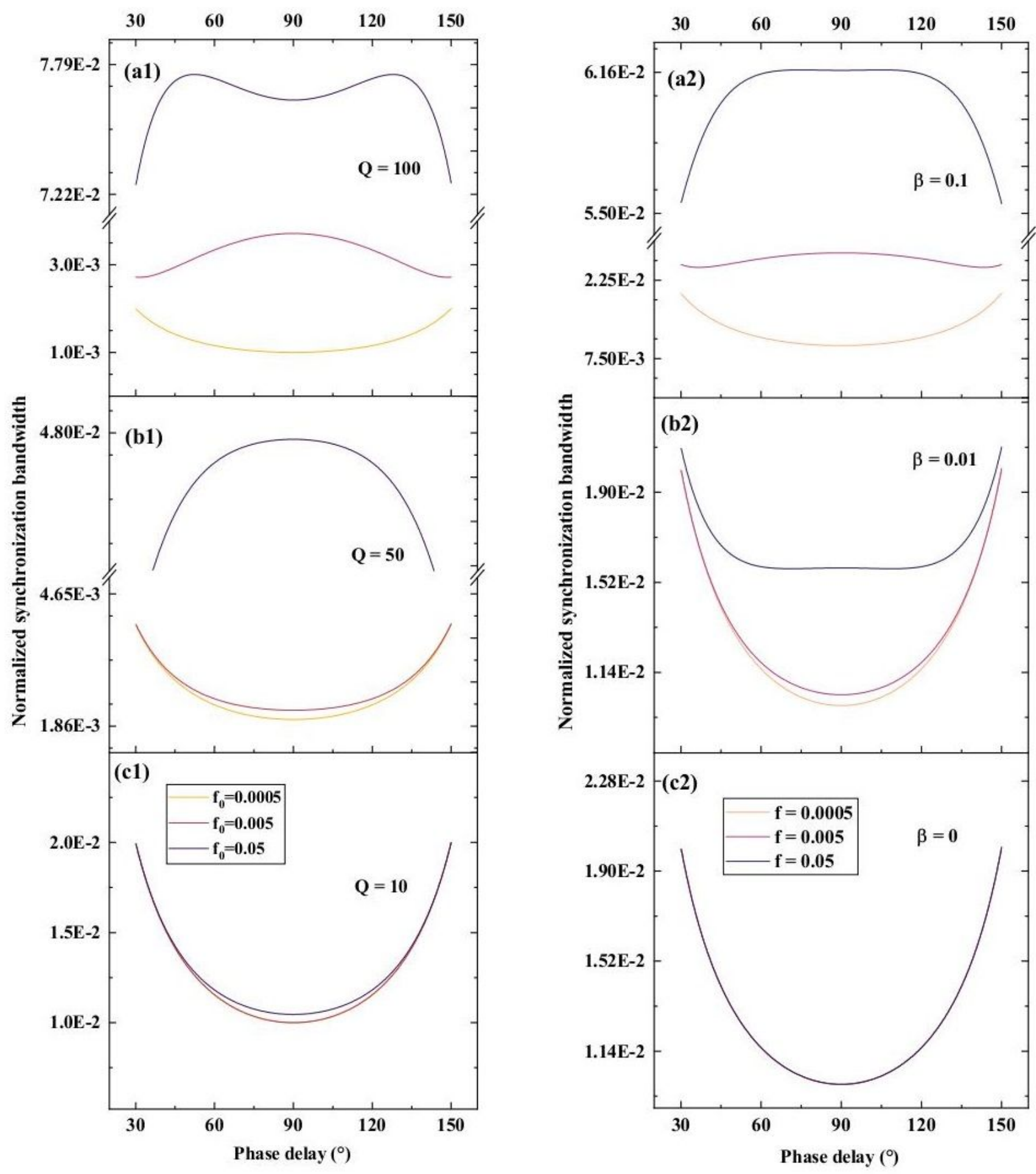

Figure 2

Please see the Manuscript PDF file for the complete figure caption 


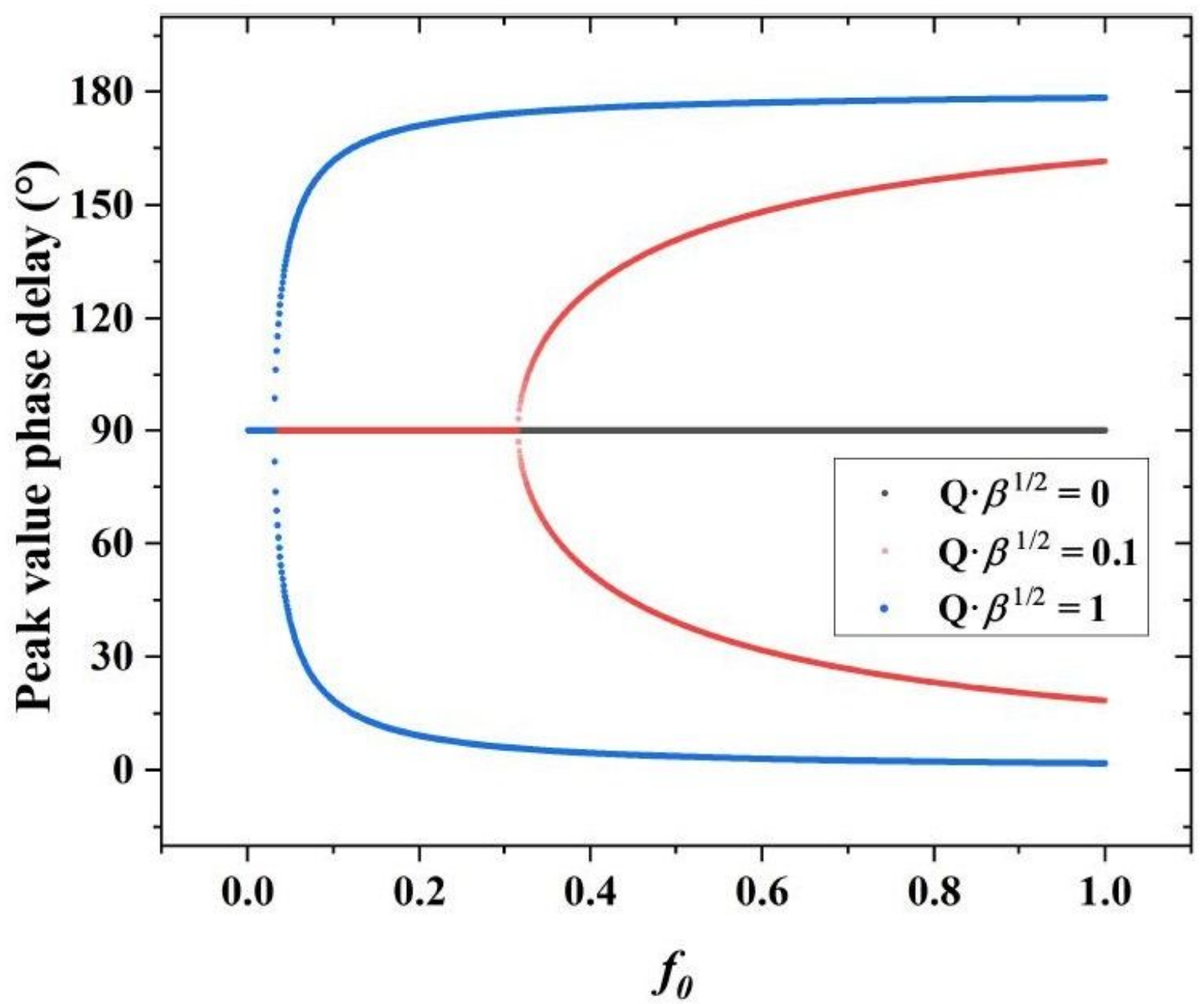

Figure 3

Please see the Manuscript PDF file for the complete figure caption 
(a)

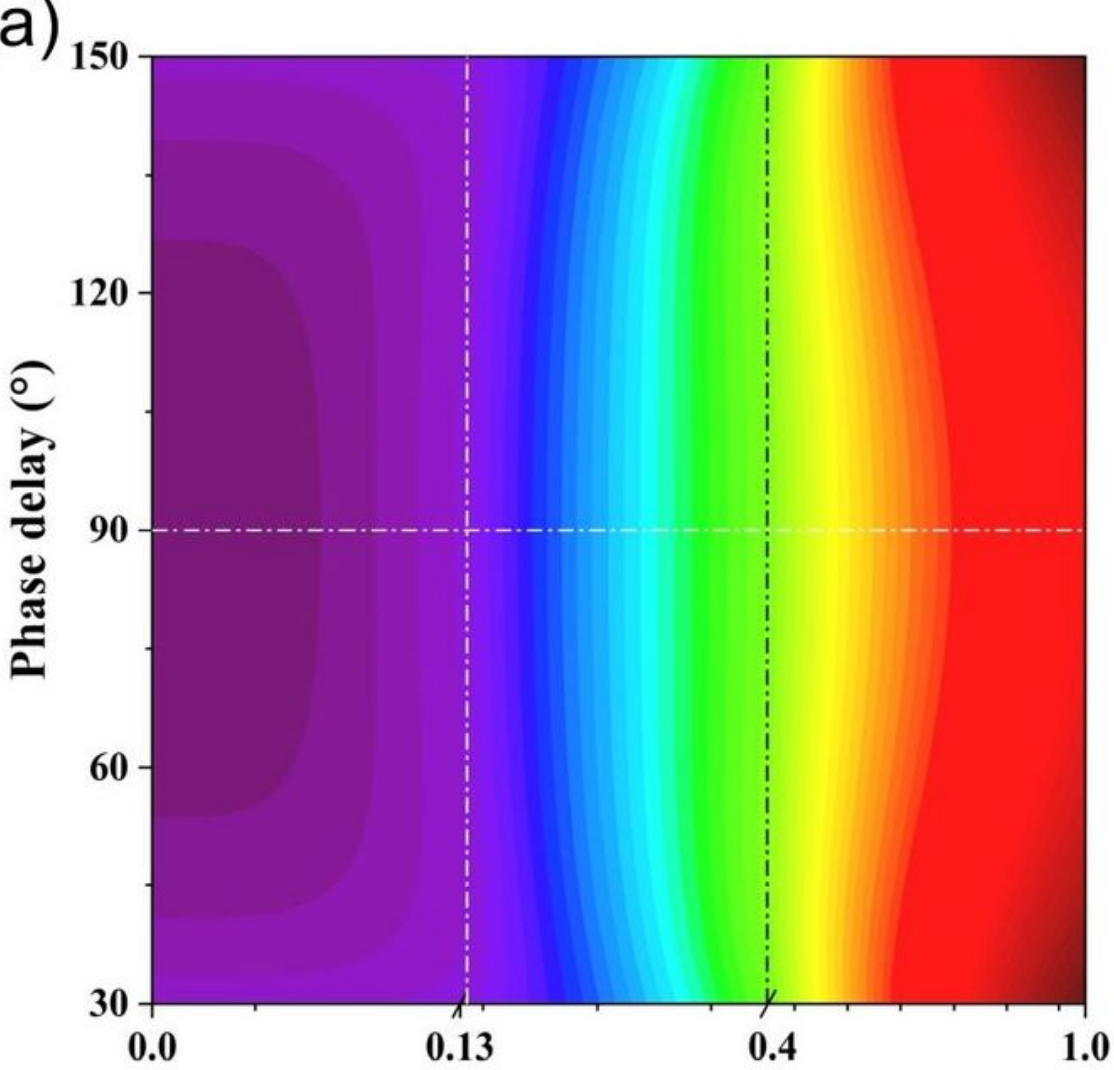

Sync-size

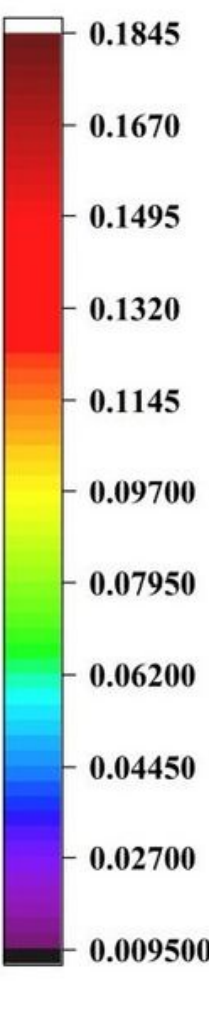

(b)

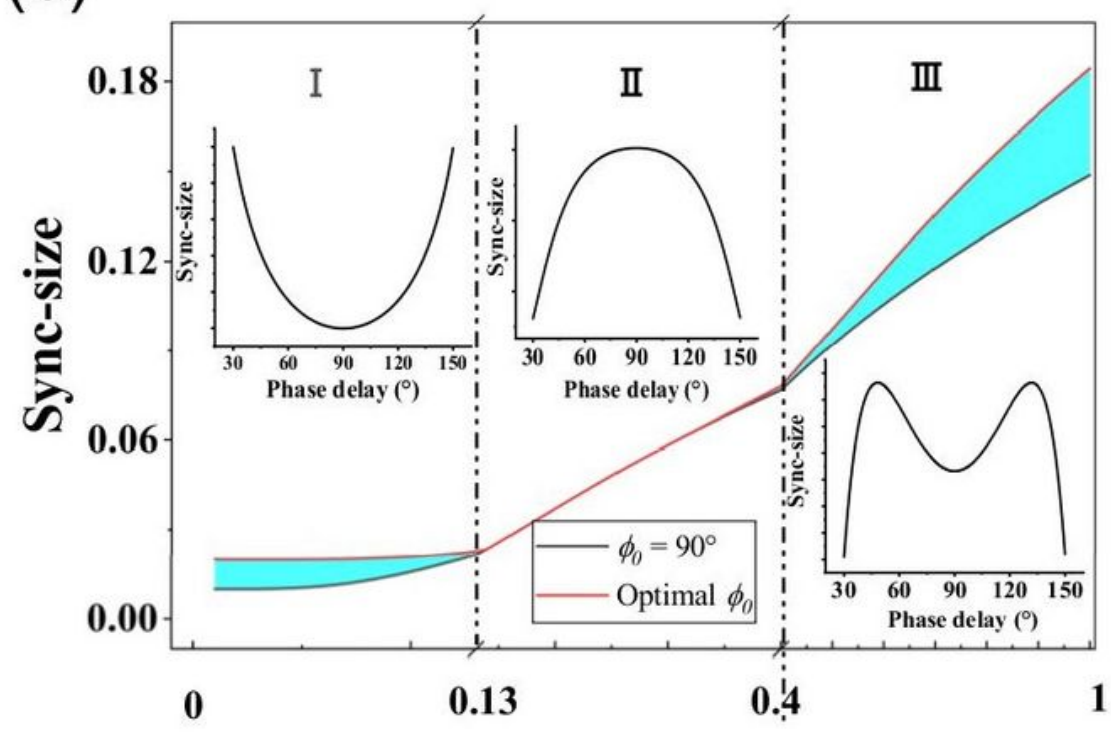

Feedback force $f_{0}$

Figure 4

Please see the Manuscript PDF file for the complete figure caption 


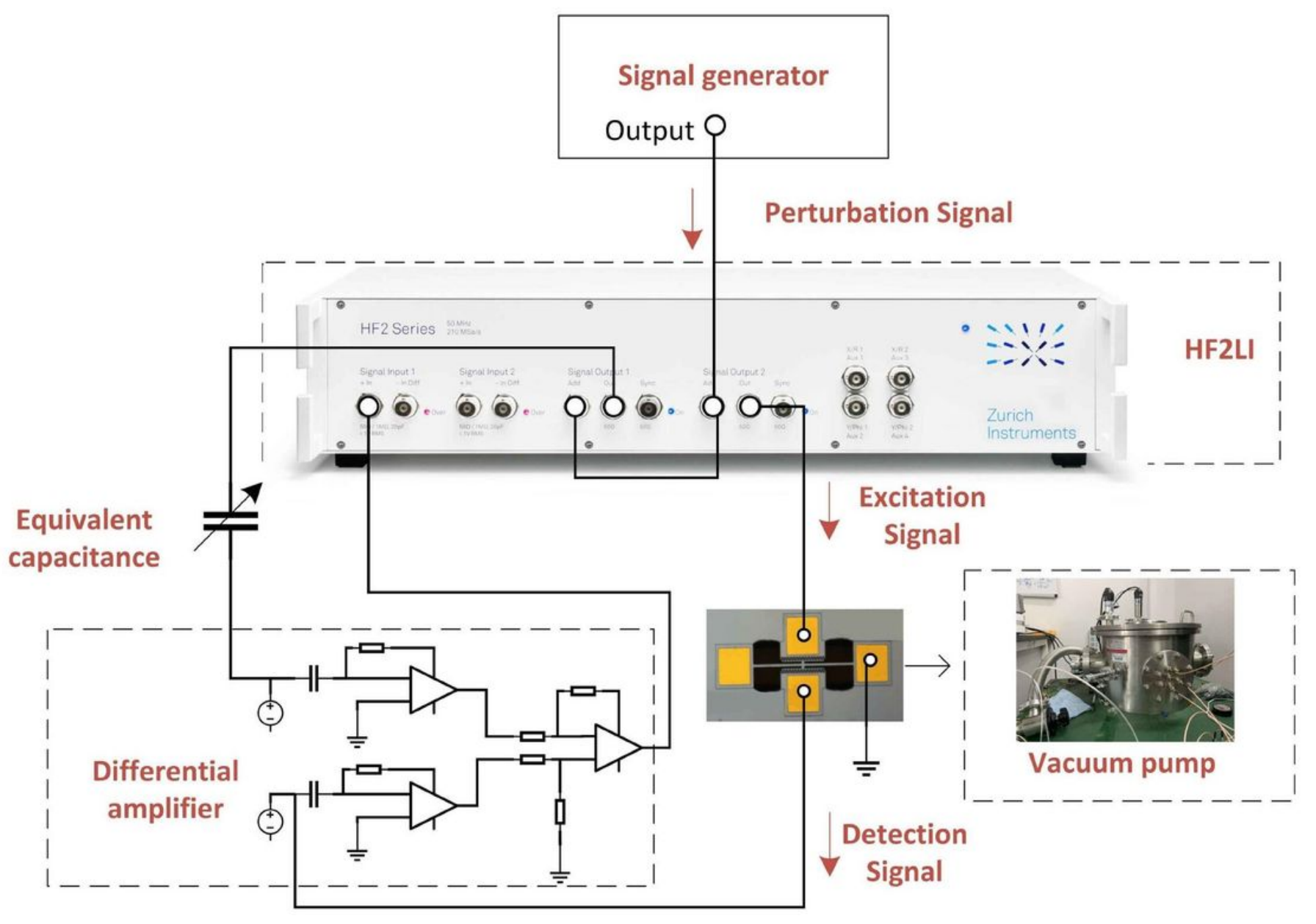

Figure 5

Please see the Manuscript PDF file for the complete figure caption 
(a)

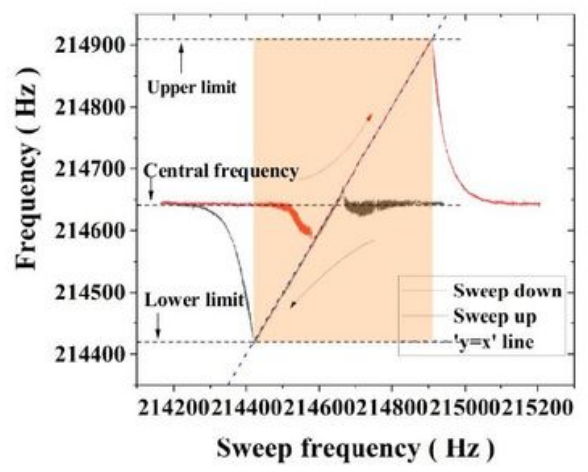

(d)

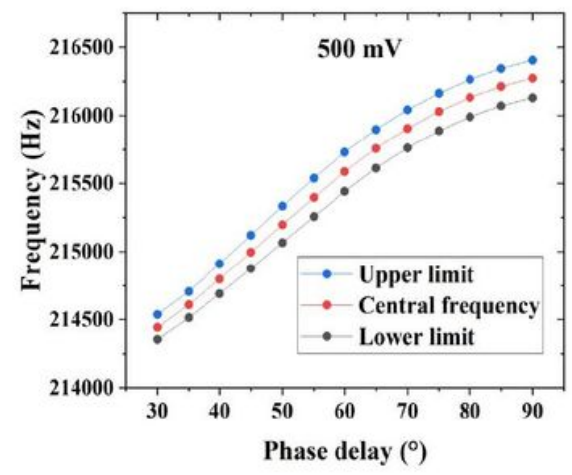

(b)

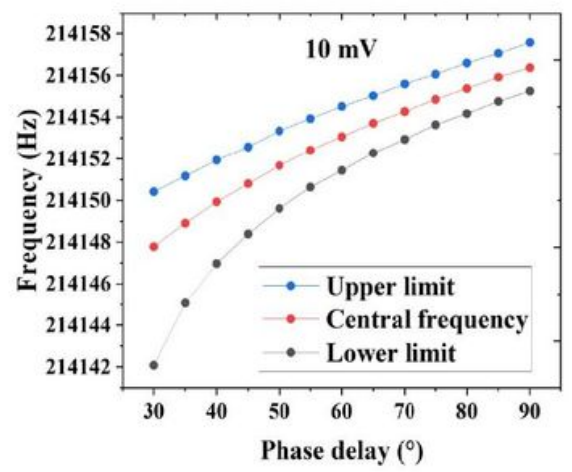

(e)

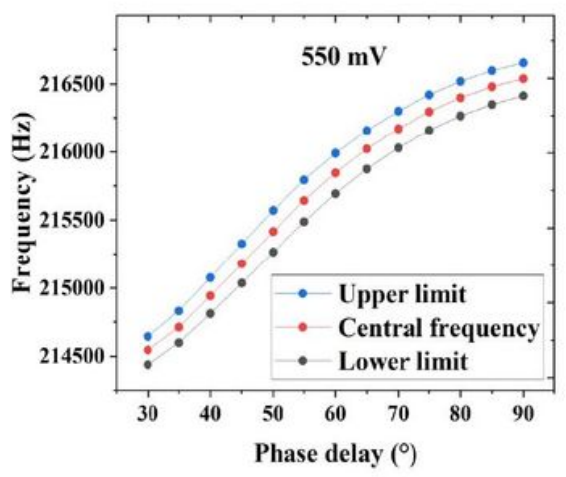

(c)

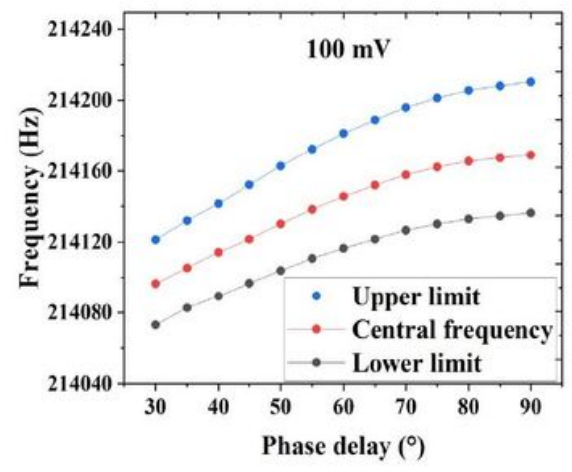

(f)

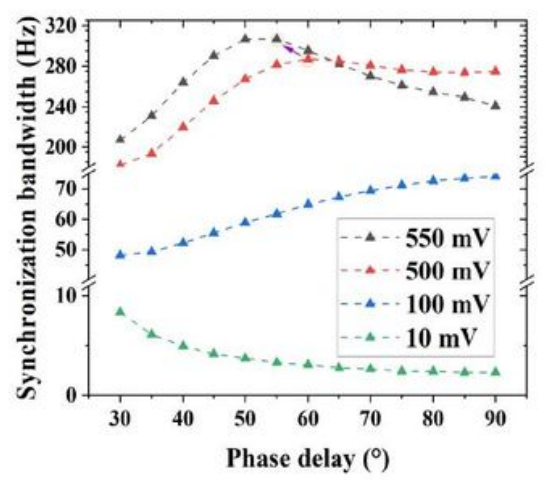

\section{Figure 6}

Please see the Manuscript PDF file for the complete figure caption 


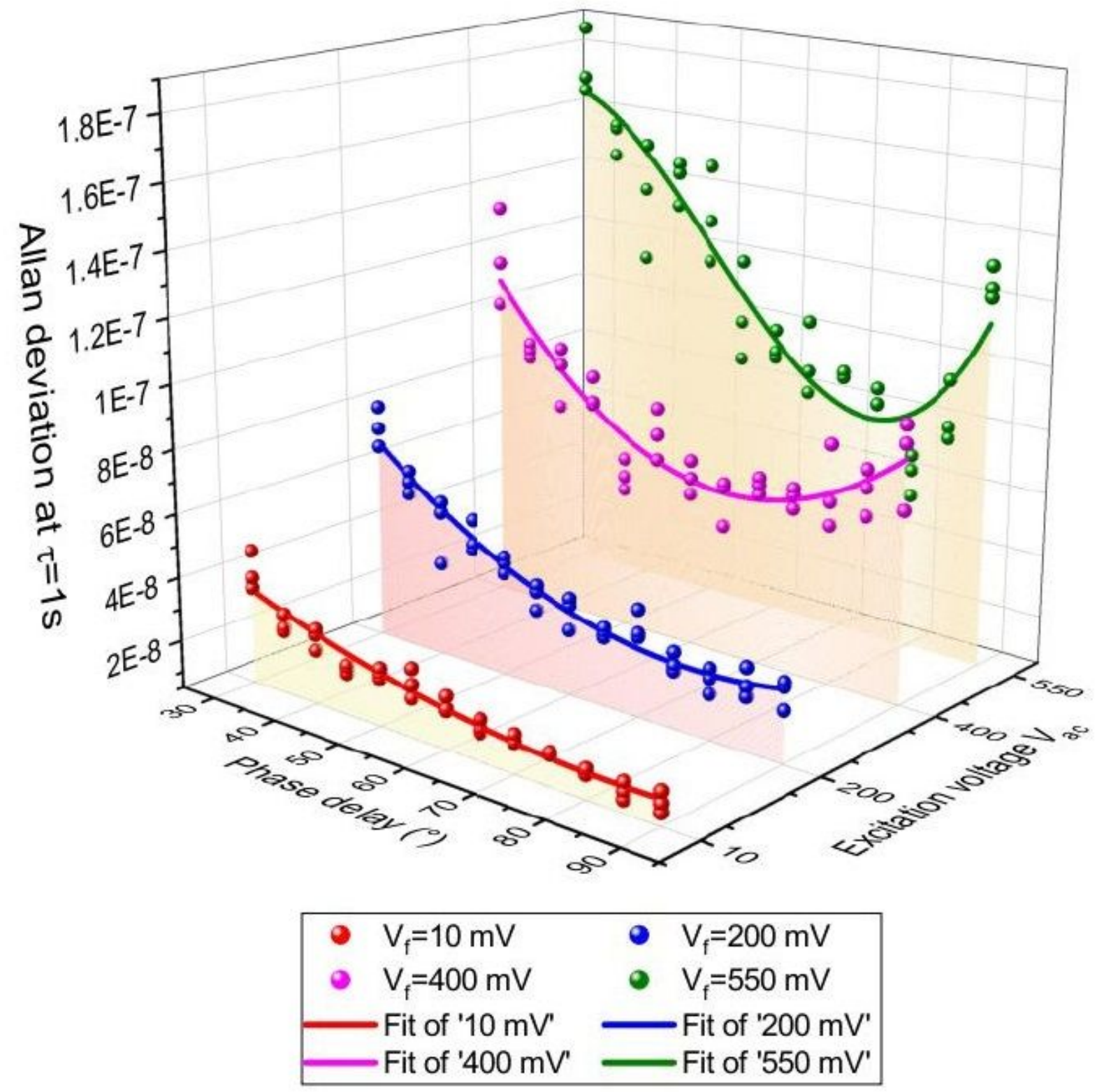

Figure 7

Please see the Manuscript PDF file for the complete figure caption 

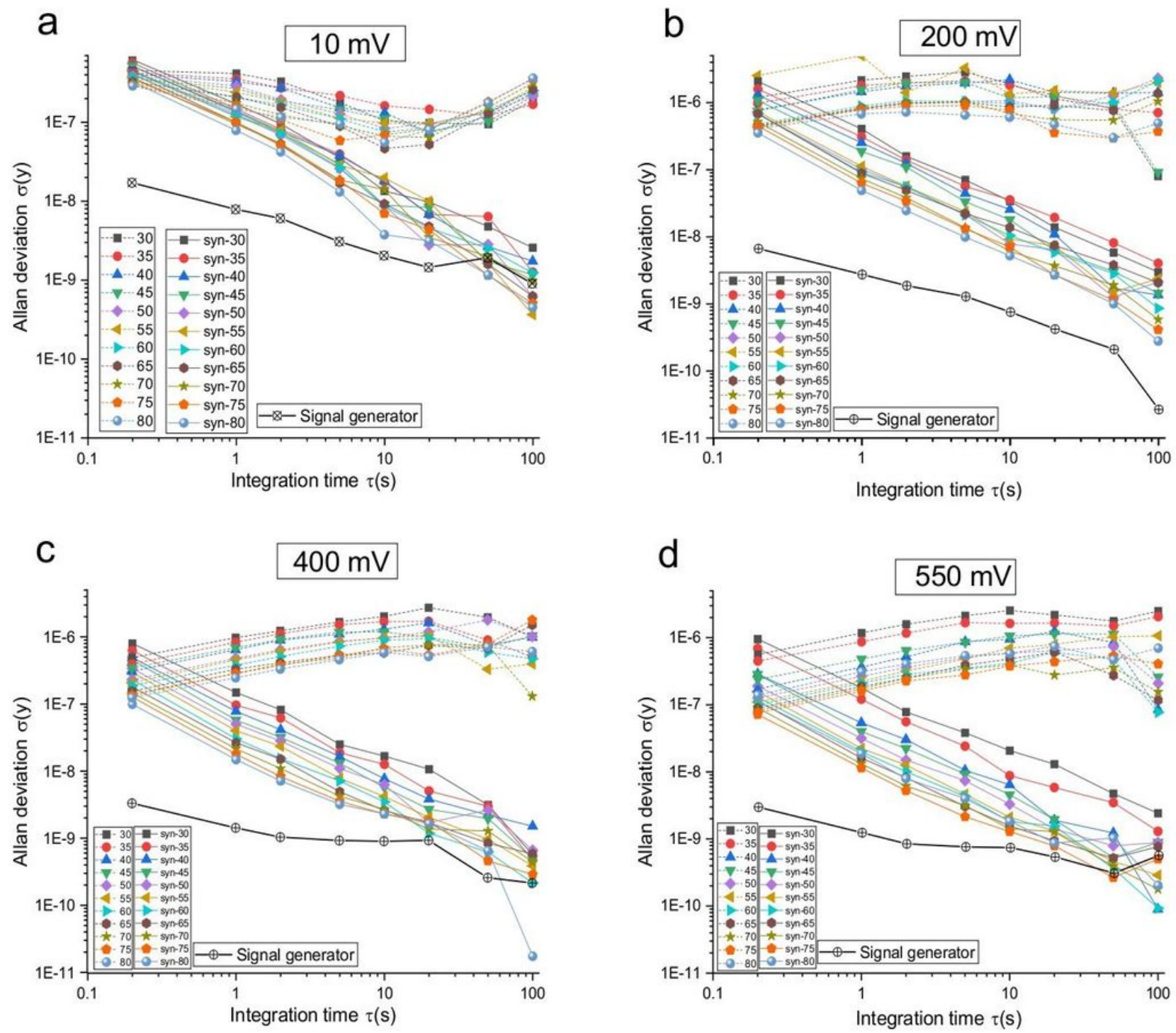

Figure 8

Please see the Manuscript PDF file for the complete figure caption 